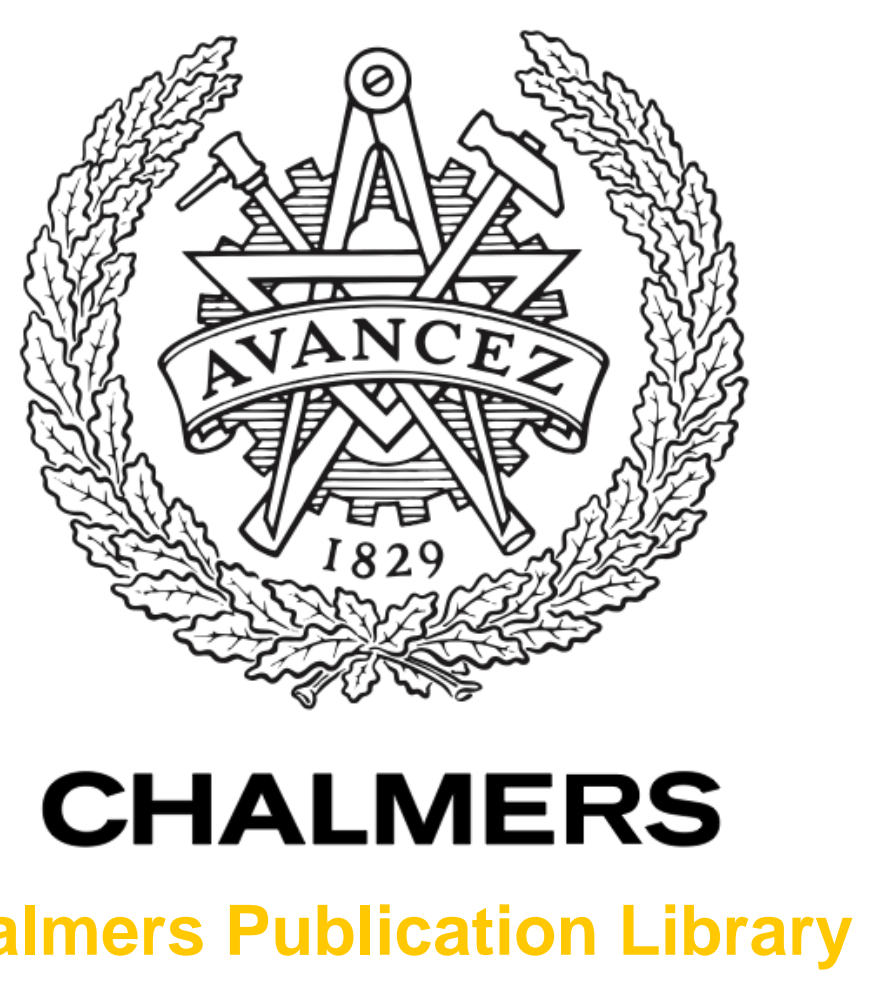

Chalmers Publication Library

Kiloparsec-Scale Dust Disks in High-Redshift Luminous Submillimeter Galaxies

This document has been downloaded from Chalmers Publication Library (CPL). It is the author's version of a work that was accepted for publication in:

Astrophysical Journal (ISSN: 0004-637X)

Citation for the published paper:

Hodge, J. ; Swinbank, A. ; Simpson, J. et al. (2016) "Kiloparsec-Scale Dust Disks in HighRedshift Luminous Submillimeter Galaxies". Astrophysical Journal, vol. 833(1),

http://dx.doi.org/10.3847/1538-4357/833/1/103

Downloaded from: http://publications.lib.chalmers.se/publication/247600

Notice: Changes introduced as a result of publishing processes such as copy-editing and formatting may not be reflected in this document. For a definitive version of this work, please refer to the published source. Please note that access to the published version might require a subscription.

Chalmers Publication Library (CPL) offers the possibility of retrieving research publications produced at Chalmers University of Technology. It covers all types of publications: articles, dissertations, licentiate theses, masters theses, conference papers, reports etc. Since 2006 it is the official tool for Chalmers official publication statistics. To ensure that Chalmers research results are disseminated as widely as possible, an Open Access Policy has been adopted.

The CPL service is administrated and maintained by Chalmers Library. 


\title{
KILOPARSEC-SCALE DUST DISKS IN HIGH-REDSHIFT LUMINOUS SUBMILLIMETER GALAXIES
}

J. A. Hodge ${ }^{1}$, A. M. Swinbank ${ }^{2,3}$, J. M. Simpson ${ }^{4}$, I. Smail ${ }^{2,3}$, F. Walter ${ }^{5}$, D. M. Alexander ${ }^{2}$, F. Bertoldi ${ }^{6}$, A. D. Biggs ${ }^{7}$, W. N. Brandt ${ }^{8,9,10}$, S. C. Chapman ${ }^{11}$, C. C. Chen $^{2}$, K. E. K. Coppin ${ }^{12}$, P. Cox $^{13}$, H. Dannerbauer ${ }^{14,15}$, A. C. EdGe $^{2}$, T. R. Greve $^{16}$, R. J. Ivison ${ }^{4,17}$, A. Karim ${ }^{6}$, K. K. Knudsen ${ }^{18}$, K. M. Menten ${ }^{6}$, H.-W. Rix ${ }^{5}$, E. Schinnerer ${ }^{5}$, J. L. Wardlow ${ }^{2}$, A. WeISS ${ }^{19}$, AND P. VAN DER WERF ${ }^{1}$

${ }^{1}$ Leiden Observatory, Leiden University, P.O. Box 9513, 2300 RA Leiden, The Netherlands; hodge@ @strw.leidenuniv.nl

${ }^{2}$ Centre for Extragalactic Astronomy, Department of Physics, Durham University, South Road, Durham, DH1 3LE, UK ${ }^{3}$ Institute for Computational Cosmology, Durham University, South Road, Durham, DH1 3LE, UK

${ }^{4}$ Institute for Astronomy, University of Edinburgh, Blackford Hill, Edinburgh EH9 3HJ, UK

${ }^{5}$ Max-Planck Institut für Astronomie, Königstuhl 17, D-69117 Heidelberg, Germany

${ }^{6}$ Argelander-Institute of Astronomy, Bonn University, Auf dem Hügel 71, D-53121 Bonn, Germany

${ }^{7}$ European Southern Observatory, Karl-Schwarzschild Strasse 2, D-85748 Garching, Germany

${ }^{8}$ Department of Astronomy and Astrophysics, 525 Davey Lab, The Pennsylvania State University, University Park, PA 16802, USA

${ }^{9}$ Institute for Gravitation and the Cosmos, The Pennsylvania State University, University Park, PA 16802, USA

${ }^{10}$ Department of Physics, 104 Davey Laboratory, The Pennsylvania State University, University Park, PA 16802, USA

${ }^{11}$ Department of Physics and Atmospheric Science, Dalhousie University, 6310 Coburg Road, Halifax, NS B3H 4R2, Canada

${ }^{12}$ Department of Physics, McGill University, 3600 Rue University, Montreal, QC H3A 2T8, Canada

${ }^{13}$ Joint ALMA Observatory-ESO, Av. Alonso de Cordova, 3104, Santiago, Chile

${ }_{15}^{14}$ Instituto de Astrofísica de Canarias, Vía Láctea s/n, E-38205, La Laguna, Tenerife, Spain

${ }^{15}$ Universität Wien, Institut für Astrophysik, Türkenschanzstrasse 17, A-1180 Wien, Austria

${ }^{16}$ University College London, Department of Physics \& Astronomy, Gower Street, London, WC1E 6BT, UK

${ }^{17}$ UK Astronomy Technology Center, Science and Technology Facilities Council, Royal Observatory, Blackford Hill, Edinburgh EH9 3HJ, UK

${ }^{8}$ Department of Earth and Space Sciences, Chalmers University of Technology, Onsala Space Observatory, SE-43992 Onsala, Sweden

${ }^{19}$ Max-Planck Institut für Radioastronomie, Auf dem Hügel 69, D-53121 Bonn, Germany

Received 2016 July 16; revised 2016 September 29; accepted 2016 October 9; published 2016 December 9

\begin{abstract}
We present high-resolution (0". 16) $870 \mu \mathrm{m}$ Atacama Large Millimeter/submillimeter Array (ALMA) imaging of 16 luminous $\left(L_{\mathrm{IR}} \sim 4 \times 10^{12} L_{\odot}\right)$ submillimeter galaxies (SMGs) from the ALESS survey of the Extended Chandra Deep Field South. This dust imaging traces the dust-obscured star formation in these $z \sim 2.5$ galaxies on $\sim 1.3 \mathrm{kpc}$ scales. The emission has a median effective radius of $R_{e}=0 . \prime 24 \pm 0 . \prime 02$, corresponding to a typical physical size of $R_{e}=1.8 \pm 0.2 \mathrm{kpc}$. We derive a median Sérsic index of $n=0.9 \pm 0.2$, implying that the dust emission is remarkably disk-like at the current resolution and sensitivity. We use different weighting schemes with the visibilities to search for clumps on 0 "' $12(\sim 1.0 \mathrm{kpc})$ scales, but we find no significant evidence for clumping in the majority of cases. Indeed, we demonstrate using simulations that the observed morphologies are generally consistent with smooth exponential disks, suggesting that caution should be exercised when identifying candidate clumps in even moderate signal-to-noise ratio interferometric data. We compare our maps to comparable-resolution Hubble Space Telescope $H_{160}$-band images, finding that the stellar morphologies appear significantly more extended and disturbed, and suggesting that major mergers may be responsible for driving the formation of the compact dust disks we observe. The stark contrast between the obscured and unobscured morphologies may also have implications for SED fitting routines that assume the dust is co-located with the optical/near-IR continuum emission. Finally, we discuss the potential of the current bursts of star formation to transform the observed galaxy sizes and light profiles, showing that the $z \sim 0$ descendants of these SMGs are expected to have stellar masses, effective radii, and gas surface densities consistent with the most compact massive $\left(M_{*} \sim 1-2 \times 10^{11} M_{\odot}\right)$ earlytype galaxies observed locally.
\end{abstract}

Key words: galaxies: evolution - galaxies: formation - galaxies: high-redshift - galaxies: starburst submillimeter: galaxies

\section{INTRODUCTION}

How high-redshift galaxies formed their stars remains an open question. Deep (rest-frame) UV/optical surveys have yielded large samples of high-redshift $(z \sim 1.5-3.5)$ starforming galaxies selected based on magnitude/color properties (BM/BX, BzK; e.g., Daddi et al. 2004, 2007a, 2007b; Steidel et al. 2004), the study of which has provided a basic picture of their formation. In particular, studies of the ionized gas kinematics in such galaxies have uncovered a high fraction of large rotating disks among the massive, optically bright systems (e.g., Förster Schreiber et al. 2006; Shapiro et al. 2008; Swinbank et al. 2012). These studies suggest that secular processes within star-forming galaxies are driving their gas and stars into the central regions, building up exponential disks and massive bulges without the need for major mergers (e.g., Elmegreen et al. 2008; Genzel et al. 2008; Dekel et al. 2009, 2013; Bournaud 2016).

The most luminous galaxies at high-redshift are the dusty star-forming galaxies originally detected in the submillimeter and known as submillimeter galaxies (SMGs; e.g., Blain et al. 2002; Solomon \& Vanden Bout 2005; Carilli \& Walter 2013; Casey et al. 2014). Their large luminosities $\left(L_{\mathrm{IR}}>10^{12-13} L_{\odot}\right.$, qualifying them as ultra- or even hyperluminous infrared galaxies) make them easier to observe in the distant universe, in principle, though whether their starformation process differs from less extreme galaxies is still 
debated. The canonical picture is that the majority of SMGs are scaled-up ultra-luminous infrared galaxies (ULIRGs; Sanders \& Mirabel 1996) - i.e., starburst-dominated major mergers (e.g., Narayanan et al. 2010); though, non-cosmological hydrodynamic simulations have suggested that SMGs could be a heterogeneous population: a mix of pre-merger pairs of disk galaxies, merger-induced starbursts, and isolated gas-rich disk galaxies undergoing a secular burst (e.g., Hayward et al. 2011, 2012). Still other models posit that the submillimeter-luminous phase is long-lived and associated with the bombardment of a central halo by numerous sub-halos in early universe proto-clusters (Narayanan et al. 2015). Finally, some models propose that SMGs may simply represent the most massive extension of the normal $z>2$ star-forming galaxy population (e.g., Kereš et al. 2005, 2009a, 2009b; Davé et al. 2010). This last theory may be at odds with claims that normal $(\mathrm{BM} / \mathrm{BX}, B z K)$ high-redshift star-forming galaxies seem to follow a different sequence than SMGs on the $M_{\text {gas }} / L_{\text {IR }}$ plane (e.g., Daddi et al. 2010; Genzel et al. 2010; Hodge et al. 2015; although see Ivison et al. 2011).

In order to better understand how SMGs fit into the larger evolutionary picture - and, more broadly, how star formation occurred in high-redshift galaxies in general-resolved observations of the spatial distribution of the star formation are essential. However, studies based solely in the (rest-frame) optical/UV (e.g., Chapman et al. 2003b, 2005; Swinbank et al. 2010a; Chen et al. 2015) must contend with dust obscuration, which can make such emission challenging to detect in the most highly star-forming galaxies, and where patchy reddening could potentially affect the apparent morphology, particularly in the rest-frame UV. Some studies therefore use the Plateau de Bure Interferometer and Karl G. Jansky Very Large Array to target radio synchrotron emission, a potential proxy for star formation, or molecular line emission (CO), which traces the gas reservoirs required to fuel star formation, at sub-arcsecond resolution ( $\gtrsim 0$ ". 2; e.g., Bothwell et al. 2010; Engel et al. 2010; Tacconi et al. 2010, 2013; Hodge et al. 2012, 2013a; Genzel et al. 2013; Aravena et al. 2014; Bolatto et al. 2015; Miettinen et al. 2015). The molecular gas studies, in particular, reveal large clumpy disks in both the more "normal" high-redshift galaxies and even in some SMGs (Hodge et al. 2012), in apparent agreement with claims of $\sim$ kiloparsec-scale star-forming regions in high-redshift galaxies from the rest-frame optical/UV (e.g., Elmegreen et al. 2004; Förster Schreiber et al. 2011; Guo et al. 2012, 2015) and $\mathrm{H} \alpha$ line emission (Genzel et al. 2011). Such massive kiloparsec-scale clumps are thought to form insitu by gravitational instability due to the gas-richness of these high-redshift galaxies (e.g., Dekel et al. 2009; Bournaud et al. 2014). Moreover, molecular gas observations can also provide valuable information on the kinematics of the systems. For example, based on observations of continuum and various $\mathrm{CO}$ transitions (up to CO[7-6]) in a sample of 12 SMGs, Engel et al. (2010) suggested that practically all SMGs are major mergers. However, such studies have been very expensive observationally, and in many cases at best marginally resolve the sources (see Carilli \& Walter 2013 for a review).

A more direct way to trace the obscured star-forming regions in high-redshift galaxies is through observations of the dust continuum emission in the rest-frame far-infrared (FIR), corresponding to observed submillimeter wavelengths for sources at $z>1$. The FIR dust continuum is dominantly powered by recently formed, massive stars, making it an excellent tracer of the bolometric luminosity-and thus star formation-in dusty starbursts such as SMGs. While the resolution achievable by early submillimeter interferometric observations (e.g., Dannerbauer et al. 2008; Wang et al. 2011; Smolčić et al. 2012; Hodge et al. 2013b) was too poor $\left(>1^{\prime \prime}\right)$ to sufficiently resolve high-redshift galaxies except for in a handful of cases (e.g., Younger et al. 2008; Hodge et al. 2012), recently, there have been some first attempts to constrain the sizes of larger samples of SMGs - as well as massive dusty star-forming galaxies selected as likely progenitors of $z \sim 2$ compact quiescent galaxies-in the submillimeter (e.g., Ikarashi et al. 2015; Simpson et al. 2015b; Barro et al. 2016), revealing compact $\left(R_{e} \sim 1 \mathrm{kpc}\right)$ dusty starbursts. However, how this star formation is distributed within the sources-e.g., whether it lies in clumpy disks or is strongly centrally peaked due to the violent and dissipative collapse expected from major merger remnants (Bournaud et al. 2011) -is still unknown. Moreover, only in rare cases of gravitational magnification (Swinbank et al. 2010b; Hatsukade et al. 2015) or case studies of single extreme sources (Hodge et al. 2015; Oteo et al. 2016) have individual star-forming regions in an SMG-or any highredshift galaxy - been potentially resolved in the FIR. While seemingly consistent with the kiloparsec-scale clumps observed in the rest-frame optical/UV and $\mathrm{H} \alpha / \mathrm{CO}$ line emission, the reality of these low-signal-to-noise ratio $(\mathrm{S} / \mathrm{N})$ "clumps" - which are argued to play a key role in high-redshift galaxy formation and evolution - has not yet been confirmed.

With Atacama Large Millimeter/submillimeter Array (ALMA), the situation is now fundamentally changed. The long baselines and large number of antennas make it possible to resolve the star-forming regions in galaxies on scales of $\lesssim 1 \mathrm{kpc}$, similar to the resolution achievable for nearby galaxies with Herschel, and at a sensitivity sufficient to map the morphology of the emission. We therefore used ALMA to conduct high-resolution $(\sim 0$ ". 16 FWHM) Band 7 (344 GHz) mapping of the (rest-frame) FIR-continuum in 17 SMGs selected from our ALMA Cycle 0 compact configuration survey of single-dish $344 \mathrm{GHz}$ LABOCA sources detected in the Extended Chandra Deep Field South (ECDFS) by Weiß et al. (2009), constituting the largest, most homogenous, and highest-sensitivity sample of interferometrically observed SMGs to date (ALESS; Hodge et al. 2013b; Karim et al. 2013).

We begin in Section 2 with the details of the observations. Our results are presented in Section 3, followed by a discussion in Section 4. We summarize our conclusions in Section 5. Where applicable, we assume a concordance, flat $\Lambda \mathrm{CDM}$ cosmology of $\mathrm{H}_{0}=71 \mathrm{~km} \mathrm{~s}^{-1} \mathrm{Mpc}^{-1}, \Omega_{\Lambda}=0.73$, and $\Omega_{M}=0.27$ (Spergel et al. 2003, 2007). All magnitudes are on the $\mathrm{AB}$ system.

\section{OBSERVATIONS AND DATA REDUCTION}

\subsection{Sample Selection and Observations}

The ALMA observations analyzed here were taken between 2015 August 11-27 as part of our rolled-over Cycle 1 Project \#2012.1.00307.S. We targeted 15 fields from our Cycle 0 ALESS survey (Hodge et al. 2013b), which itself observed 122 of the 126 single-dish-selected submillimeter sources originally detected in the LESS survey of the ECDFS (Weiß et al. 2009). The 15 fields were selected from $\sim 40$ fields that, as of the Cycle 1 proposal deadline in early 2012, had either existing or 


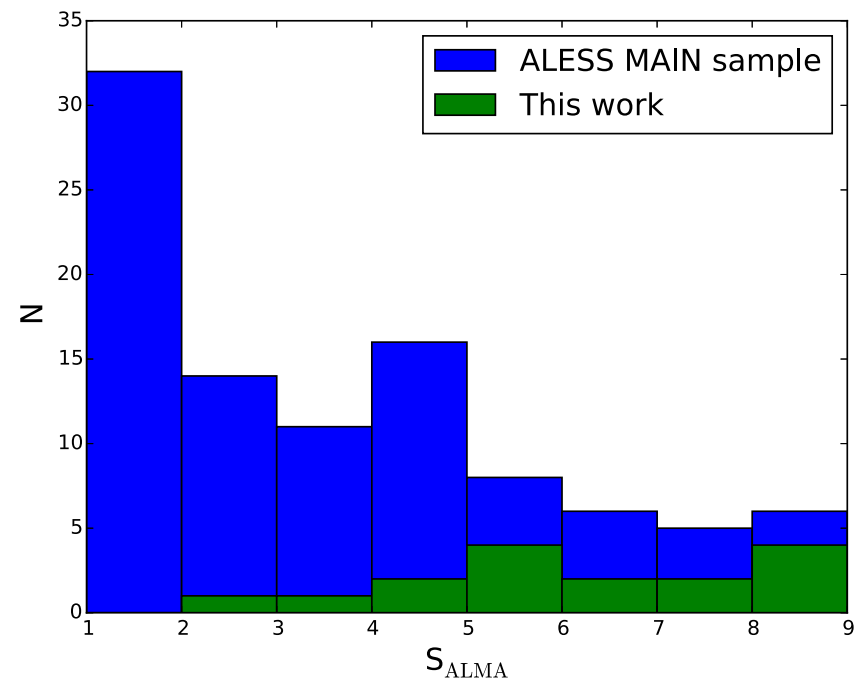

Figure 1. ALMA $870 \mu \mathrm{m}$ flux density for the high-resolution sources targeted in this paper compared to the entire ALESS MAIN sample. The flux densities have been corrected for the effect of flux boosting - see Section 2.3. The highresolution targets of this study were chosen from the randomly selected $H S T$ covered fields and preferentially target the brighter ALMA SMGs in this field.

forthcoming deep Hubble Space Telescope (HST) observations through CANDELS or our Cycle 20 HST program (Chen et al. 2015). Specifically, we selected the fields containing the submillimeter-brightest ALMA SMGs from the HST-covered fields, which were themselves randomly selected. Although some of the ALESS SMGs may be marginally resolved in the $\sim 1$ ". 6 (FWHM) Cycle 0 data along one or more axes (and only one source definitively so; Hodge et al. 2013b), no selection was made on source extent or morphology in the ALMA or $H S T$ images so as to avoid biasing the results. Four of the SMGs are associated with X-ray sources (ALESS 17.1, 45.1, 67.1, and 73.1; Wang et al. 2013). The flux density distribution for the sources targeted in this program compared with that for the entire ALESS Cycle 0 sample is shown in Figure 1, where we see that the sources targeted in this study are slightly brighter than the average SMGs.

As in our Cycle 0 ALESS program, we observed all fields with ALMA's Band 7 centered at $344 \mathrm{GHz} / 870 \mu \mathrm{m}$ to facilitate direct comparison of the measured flux densities. We utilized the "single continuum" spectral mode, with $4 \times 128$ dual polarization channels over the $8 \mathrm{GHz}$ bandwidth. At this frequency, ALMA has a 17!' 3 primary beam (FWHM).

Three fields (LESS 1, 15 and 67) contained multiple SMGs detected in the Cycle 0 MAIN ALESS catalog at 1 ." 6 resolution, and four fields contained SMGs from the Cycle 0 SUPPLEMENTARY catalog in addition to the primary source(s) from the MAIN catalog (Hodge et al. 2013b). In all cases except for LESS 1, the ALMA beam was centered on the brightest Cycle 0 ALESS source in the field in order to maximize sensitivity for the high-resolution observations. As a result, the majority of the Cycle 0 SUPPLEMENTARY sources fall outside the coverage of the ALMA beam. The observations presented here thus include 18 SMGs from the Cycle 0 MAIN catalog and one SMG from the Cycle 0 SUPPLEMENTARY catalog, or 19 SMGs in total (within the 17!' 3 FWHM of the primary beam).

The ALMA observations were requested in the C32-6 configuration and carried out with 46 antennas in an extended configuration (minimum baseline of $\sim 15 \mathrm{~m}$, maximum baseline of $\sim 1.6 \mathrm{~km}$ ). The phase, flux, and bandpass calibrators were
J0348-2749, J0334-401, and J0522-3627, respectively, and the total integration time on each of the target fields was approximately eight minutes. The phase stability/weather conditions were good, with a median PWV at the zenith of $\sim 0.7 \mathrm{~mm}$.

\subsection{Data Reduction and Imaging}

The ALMA data were reduced using the Common Astronomy Software Application ${ }^{20}$ (CASA) version 4.3.1. The delivered reduction produced $u v$-data products of high quality and was therefore used without further modifications. The $u v$ data were imaged using CASA version 4.3.1, with subsequent analyses carried out in CASA version 4.5.0.

Imaging was carried out using the CLEAN algorithm with a variety of different weightings and $u v$-taperings to explore the extent to which the sources were resolved by the observations and the total flux densities were recovered (see Section 2.3). The (compact configuration) Cycle 0 data were not co-added to the new data given the much poorer data quality and (in some cases) offset pointing centers. For the untapered maps, multi-scale CLEAN (Cornwell 2008) was employed using scales of $\left[0^{\prime \prime}, 0 . \prime 3,0 ! \prime 6,1.2\right]$. While the largest scale was set to approximately encompass the largest coherent structure visible in the maps, we found that the specific number and distribution of these scales did not significantly affect the results, in agreement with other studies (e.g., Rich et al. 2008).

All maps were cleaned interactively using 1." 5 circular regions around sources with emission in clear excess $(\sim 4-5 \sigma)$ of the residuals. These sources were cleaned down to $\sim 2.5 \sigma$, a process that typically required one to five major clean cycles of 50 iterations each. The resulting images are 25 ." 6 per side and have a pixel scale of $0 . \prime 02$, and the naturally weighted maps achieve a typical synthesized beam of 0 !" $17 \times 0$ " 15 and rms noise of $\sim 64 \mu \mathrm{Jy}$ beam $^{-1}$, corresponding to a rest-frame brightness temperature of $T_{B}=0.09 \mathrm{~K}$ at $z \sim 2.5$. A set of maps was also produced using Briggs weighting with a robust parameter of $R=-0.5$, resulting in a resolution of 0 ." $12 \times 0$." 11 and typical rms noise values of $\sim 130 \mu \mathrm{Jy}_{\text {beam }}{ }^{-1}$. We did not attempt to self-calibrate the data. The absolute flux calibration has an uncertainty of $\sim 10 \%$, and this uncertainty is not included in the error bars for individual source flux densities.

Of the 19 Cycle 0 SMGs targeted by this project, 16 were detected in the new ALMA data at very high $\left(\mathrm{S} / \mathrm{N}_{\text {peak }}>10 \sigma\right)$ significance, allowing us to investigate the distribution of their dusty star formation. These SMGs have flux densities ranging from $\mathrm{S}_{870 \mu \mathrm{m}}=3.4-9.0 \mathrm{mJy}$ in our Cycle 0 data $(\sim 1 . " 6$ FWHM). Of the three remaining SMGs, one (ALESS 1.3) was detected at lower significance $\left(\mathrm{S} / \mathrm{N}_{\text {peak }}<10 \sigma\right)$ and two others (ALESS 15.3 and 67.2) were undetected. These sources had flux densities of 2.0 and $1.7 \mathrm{mJy}$ (corresponding to $\mathrm{S} / \mathrm{N}$ values of 3.8 and 4.2) in the Cycle 0 catalog, respectively, and based on the multi-wavelength data presented in Simpson et al. (2014), it is possible that ALESS 15.3 was spurious and ALESS 67.2 has been resolved out (see C. C. Chen et al. 2016, in preparation). In Figure 2, we show image cutouts for each of the 16 strongly detected SMGs in the naturally weighted maps $(0$ " $17 \times 0$." $15 \mathrm{FWHM}$ resolution $)$, where the extended nature of the SMGs is readily apparent. These sources span a redshift range of $z=0.76-4.95$, with a median redshift $(z=2.6 \pm 0.5)$ and infrared luminosity

\footnotetext{
${ }^{20}$ http://casa.nrao.edu
} 


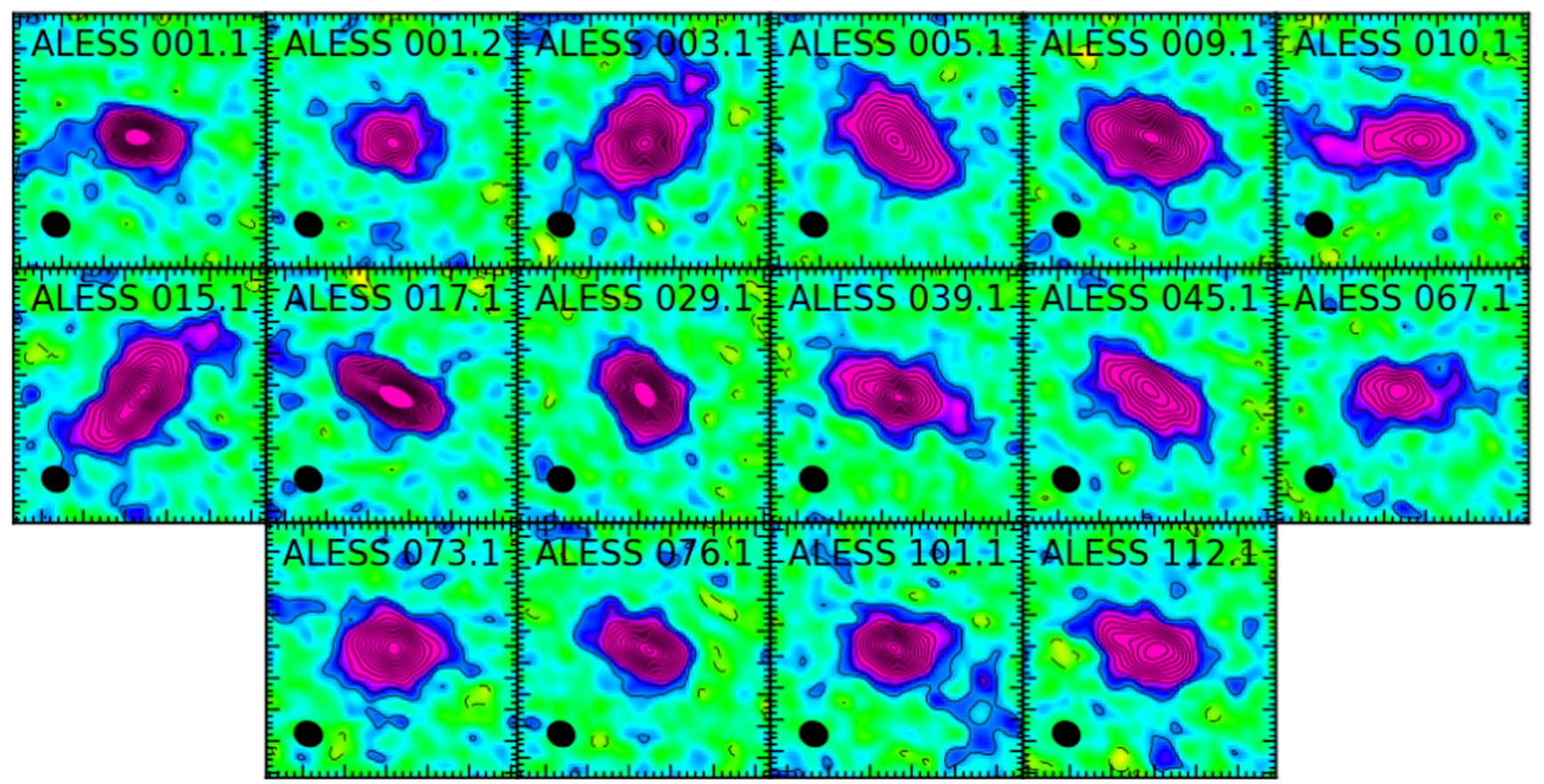

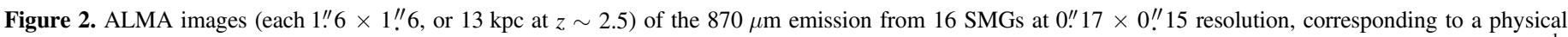

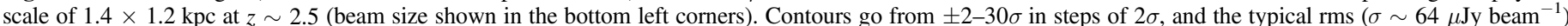

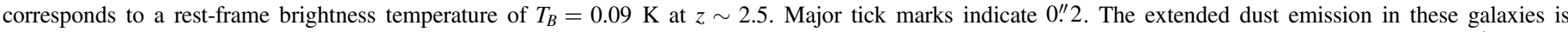

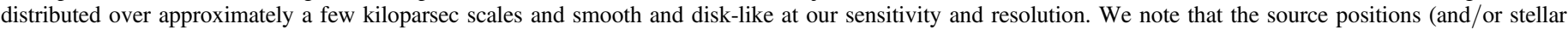
environments) of ALESS 5.1 and 10.1 suggest these sources are potentially weakly lensed (see also Figure 11 and Section 3.2).

$\left(L_{\mathrm{IR}}=3.6 \pm 0.9 \times 10^{12} L_{\odot}\right) \quad$ consistent with the sample as a whole $\left(z=2.5 \pm 0.2\right.$ and $L_{\mathrm{IR}}=3.0 \pm 0.3 \times 10^{12} L_{\odot}$; Simpson et al. 2014; Swinbank et al. 2014).

\subsection{Recovered Flux Density}

In order to test whether our new, higher-resolution ALMA images recover all of the flux density from the sources, we compared the images made at various spatial resolutions with the results obtained in Cycle 0 using a more compact configuration. The Cycle 0 flux densities were taken from Hodge et al. (2013b) and have been corrected for the effect of flux boosting (e.g., Simpson et al. 2015a), which is a statistical enhancement, on average, of the measured fluxes for populations where fainter sources far outnumber the brighter ones. In such cases, every measurement is more likely to result from one of many fainter sources than from one of few brighter ones relative to the measurement, and the effect is most pronounced for low $\mathrm{S} / \mathrm{N}$ detections. For the new data imaged at a particular resolution, we calculated the flux density recovered by masking the emission below $2 \sigma$. For the untapered data, we then used the masks from the next lowest resolution to mask the higherresolution images further (e.g., 0." 3 masks for the 0" 17 images; 0 . 17 masks for the 0 ". 12 images). This combination of steps allowed us to isolate $>2 \sigma$ contiguous emission associated with each detected source in an automated way, which we then summed using an aperture of radius $3 \times b_{\text {maj }}$, where $b_{\text {maj }}$ is the FWHM (major axis) of the synthesized beam at that resolution.

Figure 3 shows the flux density recovered as a function of angular resolution (expressed as a fraction of the Cycle 0 flux density) for individual sources and the sample median. For most sources, the recovered fraction rises steeply from the highest-resolution maps $(\sim 0$ )! 1 ; median fraction of $f=74 \pm 7 \%)$ to the naturally weighted maps $(\sim 0$.' 16 ; median

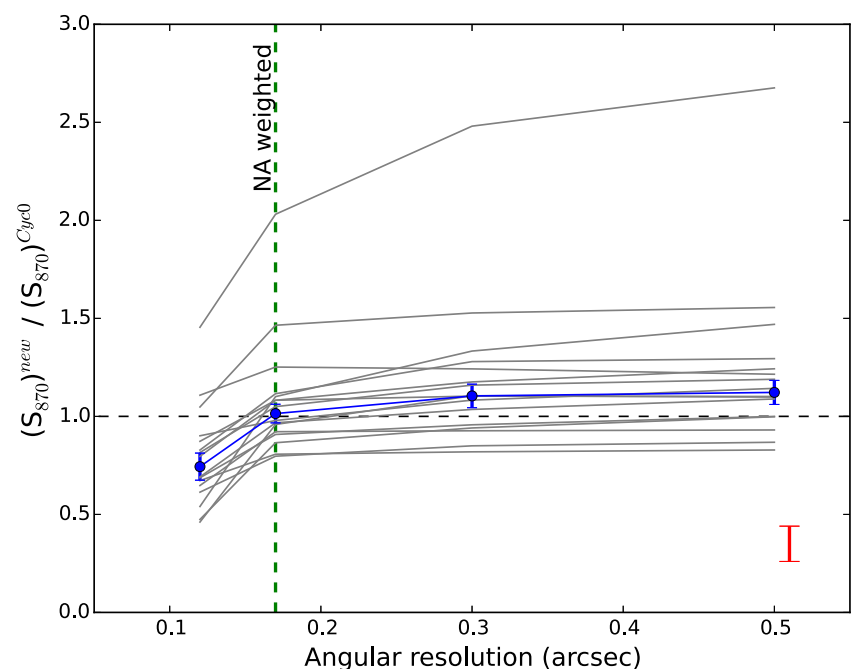

Figure 3. Fraction of the Cycle 0 flux density recovered for sources imaged at various spatial resolutions in our new study. The horizontal dashed line indicates a recovery fraction of $100 \%$ compared to the earlier, low-resolution Cycle 0 data, and the vertical dashed line indicates the resolution of the naturally weighted maps. The median recovered fraction for the sample is shown by the solid blue line, and the red error bar shows the absolute flux calibration uncertainty. The highest outlier corresponds to a source (ALESS 101.1) from a lower quality (SUPPLEMENTARY) Cycle 0 map. While the naturally weighted images may be missing a fraction $(\sim 10 \%-15 \%)$ of the emission from what is presumably a more extended component $\left(\geqslant 1^{\prime \prime}\right.$ or $\geqslant 10 \mathrm{kpc}$ ), the new ALMA observations are not formally resolving out emission, consistent with the maximum recoverable scale expected for this configuration $\left(\gtrsim 2^{\prime \prime}\right)$.

fraction of $f=101 \pm 6 \%$ ). This indicates, at face value, that the naturally weighted maps are recovering all of the flux detected in the Cycle 0 maps. However, there appears to be a potentially small increase in the recovered fraction in the $u v$ - 
Table 1

ALESS SMG Observed $870 \mu \mathrm{m}$ Dust Morphologies

\begin{tabular}{|c|c|c|c|c|c|c|c|}
\hline \multirow[t]{2}{*}{ Source ID } & \multicolumn{2}{|c|}{$\mathrm{FWHM}_{\text {maj }}{ }^{\mathrm{a}}$} & \multirow[t]{2}{*}{$b / a^{\mathrm{b}}$} & \multirow{2}{*}{$\begin{array}{c}\mathrm{PA}^{\mathrm{c}} \\
{[\mathrm{deg}]}\end{array}$} & \multirow{2}{*}{$\underset{\left[{ }^{\prime \prime}\right]}{\text { cWHirc }{ }^{\mathrm{d}}}$} & \multirow[t]{2}{*}{$R^{\mathrm{e}}$} & \multirow[t]{2}{*}{$n^{\mathrm{f}}$} \\
\hline & {$\left[{ }^{\prime \prime}\right]$} & {$[\mathrm{kpc}]$} & & & & & \\
\hline ALESS 1.1 & $0.27 \pm 0.01$ & $1.8 \pm 0.1$ & 0.5 & $80 \pm 3$ & $0.23 \pm 0.02$ & 0.16 & 1.7 \\
\hline ALESS 3.1 & $0.38 \pm 0.02$ & $2.6 \pm 0.1$ & 0.7 & $138 \pm 10$ & $0.33 \pm 0.02$ & 0.24 & 1.4 \\
\hline ALESS 5.1 & $0.50 \pm 0.03$ & $4.0 \pm 0.2$ & 0.6 & $44 \pm 3$ & $0.38 \pm 0.01$ & 0.26 & 0.7 \\
\hline ALESS 9.1 & $0.44 \pm 0.02$ & $3.0 \pm 0.1$ & 0.6 & $72 \pm 3$ & $0.35 \pm 0.01$ & 0.23 & 0.7 \\
\hline ALESS 17.1 & $0.40 \pm 0.02$ & $3.5 \pm 0.1$ & 0.3 & $62 \pm 1$ & $0.29 \pm 0.02$ & 0.20 & 0.5 \\
\hline ALESS 29.1 & $0.31 \pm 0.01$ & $2.7 \pm 0.1$ & 0.6 & $35 \pm 3$ & $0.26 \pm 0.01$ & 0.16 & 0.7 \\
\hline ALESS 39.1 & $0.47 \pm 0.03$ & $3.9 \pm 0.2$ & 0.4 & $73 \pm 4$ & $0.32 \pm 0.02$ & 0.27 & 1.2 \\
\hline ALESS 45.1 & $0.51 \pm 0.04$ & $4.3 \pm 0.3$ & 0.4 & $56 \pm 2$ & $0.37 \pm 0.01$ & 0.26 & 0.5 \\
\hline ALESS 67.1 & $0.44 \pm 0.04$ & $3.7 \pm 0.3$ & 0.5 & $89 \pm 6$ & $0.32 \pm 0.02$ & 0.25 & 1.2 \\
\hline ALESS 73.1 & $0.36 \pm 0.02$ & $2.4 \pm 0.1$ & 0.7 & $89 \pm 9$ & $0.34 \pm 0.02$ & 0.20 & 1.0 \\
\hline ALESS 76.1 & $0.33 \pm 0.02$ & $2.5 \pm 0.2$ & 0.5 & $64 \pm 3$ & $0.27 \pm 0.01$ & 0.17 & 0.6 \\
\hline
\end{tabular}

Notes.

${ }^{a}$ FWHM of the major axis derived from a two-dimensional Gaussian fit in the image plane.

b Axis ratio from the two-dimensional Gaussian fit.

${ }^{c}$ Position angle from the two-dimensional Gaussian fit.

${ }^{\mathrm{d}}$ FWHM of a one-dimensional Gaussian fit to the azimuthally averaged profile in the image plane.

e Effective (half-light) radius of the major axis from a two-dimensional Sérsic profile fit. The typical error ranges from $15 \%$ to $27 \%$.

${ }^{f}$ Sérsic index from the two-dimensional Sérsic profile fit. The typical error is in the range of $26 \%-33 \%$.

tapered data, with median fractions of $f=110 \pm 6 \%$ and $f=112 \pm 6 \%$ in the $0 . \prime 3$ and $0 . " 5$ maps, respectively. This modest excess may in part be due to the uncertainty in the overall flux calibration between the data sets, which, when taken into account, yields a median recovered fraction in the $u v$-tapered data consistent with the Cycle 0 values. Because the quality of the Cycle 0 data was much poorer (for example, the highest outlier in Figure 3 corresponds to SUPPLEMENTARY source ALESS 101.1 from a lower quality map; Hodge et al. 2013b), we conclude that the true flux densities are better determined by the (new) tapered images. This suggests that the naturally weighted images are at most missing a fraction $(\sim 10 \%-15 \%)$ of the emission from what is presumably a more extended component $\left(\gtrsim 2^{\prime \prime}\right)$. We conclude that, in general, the Cycle 1 observations do not appear to be formally resolving out emission due to the array configuration, consistent with the maximum recoverable scale expected for this configuration $\left(\gtrsim 2^{\prime \prime}\right)$. We will investigate whether the emission potentially "missing" from the naturally weighted maps has any implications for the implied galaxy sizes in Section 3.1.2.

\section{RESULTS}

\subsection{The Dust Profiles of Submillimeter Galaxies}

\subsubsection{Image Plane}

Figure 2 demonstrates that the dust-obscured star formation in these SMGs is extended on scales larger than our beam size $(0$." $17 \times 0$." 15). Following Simpson et al. (2015b), we quantified the morphology and extent of the emission by fitting each source in the image plane with three models: (1) a point source (assuming the CLEAN beam); (2) a two-dimensional Gaussian; and (3) a two-dimensional Sérsic profile. The residuals from the various fits are shown in Figure 10 in the
Appendix. The point source fit is ruled out in all cases by $>5 \sigma$ residuals. The parameters for the (deconvolved) two-dimensional Gaussian and Sérsic profile fits for all SMGs are listed in Table 1. While many of the SMGs appear elliptical, this is most likely due to inclination and optical depth effects. As such, we report the parameters for the fits along the major axis of each source, though we also quote the axis ratios from the Gaussian fits for completeness.

The median major axis size of the Gaussian fits is $\mathrm{FWHM}=0$. . $42 \pm 0 . " 04$, and the median axis ratio is $b / a=0.53 \pm 0.03$, where the errors on the median values were calculated via bootstrapping. The corresponding median physical size is $\mathrm{FWHM}=3.2 \pm 0.4 \mathrm{kpc}$. In the majority $(9 / 16)$ of the sources, there is no significant evidence (i.e., $>3 \sigma$ residuals from the Gaussian model) that the extra degree of freedom required for the Sérsic profile fits is justified. The remaining sources show 3-5 $\sigma$ residuals from the Gaussian model, indicating that the Sérsic profile is preferred. The median Sérsic profile has an index of $n=0.9 \pm 0.2$ and an effective radius of $R_{e}=0$." $24 \pm 0$ ". 02 , corresponding to a typical physical size of $R_{e}=1.8 \pm 0.2 \mathrm{kpc}$. Noting that a Gaussian fit is equivalent to a Sérsic profile fit with $n=0.5$ and $\mathrm{FWHM}=2.02 \times R_{e}$, the median Sérsic profile appears more centrally peaked than a Gaussian profile, and is consistent with an exponential disk. Only two SMGs (ALESS 1.2 and 101.1) have estimated Sérsic indices $n>2$, indicating more centrally peaked emission. The four SMGs associated with X-ray sources (ALESS 17.1, 45.1, 67.1, and 73.1) have median parameters $\left(R_{e}=0\right.$ ". $23 \pm 0$ ". $\left.02, n=0.8 \pm 0.2\right)$ consistent with the full sample.

In order to test the robustness of the derived parameters, we inserted 10,000 model sources with $\mathrm{S} / \mathrm{N}$ ratios similar to our observations into the naturally weighted maps to see how well we could recover their Sérsic parameters. The input parameters were drawn from uniform distributions with ranges 
of $n=0.2-5.0, \quad R_{e}=0.1-0.13, \quad$ and $\quad$ an axis ratio of $b / a=0.1-1.0$. We find that the input parameters are well-recovered, with systematic biases at the $\sim 1 \%$ level. The $1 \sigma$ scatter is a function of the input parameters, ranging from $15 \%$ to $27 \%$ for the effective radius and $26 \%-33 \%$ for the Sérsic index.

Finally, we create a deep composite image by combining $2^{\prime \prime}$ cutouts centered on the source centroids. Prior to the stacking, the individual sources were rotated to a common major axis. The best-fit two-dimensional Gaussian model has an FWHM of 0 ". $40 \pm 0$." 01 , consistent with the median FWHM of the individual two-dimensional Gaussian fits. The best-fit Sérsic profile has a Sérsic index of $n=1.0 \pm 0.1$ and an effective radius of $R_{e}=0.23 \pm 0$ " 05 , again indicating that the light profile of the dust emission is consistent with that of an exponential disk.

\subsubsection{Uv-plane Fits}

One way to address whether any flux "missing" from the naturally weighted images is having an impact on the source sizes measured in the image plane is to measure the sizes directly in the $u v$-plane. Figure 4 shows the $u v$-data for four isolated ALESS sources. The phase center of the new Cycle 1 data sets have been shifted to center exactly on the ALESS SMGs, and the data have then been radially averaged in bins of $75 \mathrm{k} \lambda$. Also shown are simulated profiles of smooth exponential disks $(n=1)$ with the same flux densities, effective radii, and axial ratios as those of the sources, and with added noise.

To compare these data to the low-resolution Cycle 0 observations, we applied the same procedure to the Cycle 0 data, which have also been scaled by the response of the Cycle 0 primary beam at the position of the SMG. As the majority of the SMGs are unresolved in the Cycle 0 data, only the central data point is shown. There is indeed no evidence that the Cycle 1 data are missing any emission, in agreement with Section 2.3.

We then fit the Cycle $1 u v$-profiles with two models: (1) a Gaussian and (2) a Gaussian plus a constant. The latter represents a point source (or point sources) in the image plane and was found to be necessary due to the signal evident at large $u v$-distances in the plots (particularly ALESS 17.1). We find that this point source component makes up $\lesssim 5 \%$ of the total emission in ALESS 5.1, 45.1, and 73.1, but it constitutes $15 \%$ of the emission in ALESS 17.1. This is likely caused by the large ellipticity observed in ALESS 17.1, which is nearly unresolved along its minor axis in our map, combined with the fact that the shortest spacings play a larger role in the $u v$-plane fitting. The FWHM values resulting from the Gaussian + constant model are listed in Figure 4.

These sizes can be most directly compared to onedimensional (circular) Gaussian fits from azimuthally averaged data in the image plane (Table 1). These values tend to be somewhat smaller on average than the 2D elliptical Gaussian fit values (median $\mathrm{FWHM}_{1 \mathrm{D}} / \mathrm{FWHM}_{2 \mathrm{D}}=0.79 \pm 0.07$ ), reflecting the ellipticity of the emission observed in the individual sources. When we include a point source component in the $u v$ plane model, we find that the FWHM sizes derived from fitting in the image and $u v$-planes agree (within the uncertainties). From this test and those reported in the Appendix A.1, we conclude that the sizes measured in the image plane are robust, and that they are unaffected by the presence of any potentially "missing" emission.

\subsection{Comparison to Stellar Emission}

Our SMGs were selected to have HST WFC3 imaging at comparable $(0$ ". 15$)$ resolution in one or more bands, providing a less dust-sensitive probe (than optical imaging) of the stellar distribution on approximately kiloparsec scales. We tied the astrometry of the HST images to the IRAC images, and the relative astrometry between the $H S T$ and ALMA images is expected to be accurate to $\sim 0$ "' 1 . False-color images constructed from a combination of the HST and ALMA data are shown as multi-band color images for a selection of SMGs with the most complete data in Figure 5, where a variety of stellar morphologies are observed. The $870 \mu \mathrm{m}$ contours for the full sample are overplotted on the $H_{160}$-band imaging in Figure 11. The source positions (and/or stellar environments) of ALESS 5.1 and 10.1 suggest that these sources are potentially weakly lensed. In particular, the redshifts of the nearby bright $H_{160}$-band counterparts suggest that these sources are at lower redshift, though we cannot rule out the possibility that they are mergers.

It is immediately clear from these comparisons that the obscured star formation traced by the dust emission is generally more compact than the stellar emission. To quantify this effect, the median curves of growth for the naturally weighted ALMA $870 \mu \mathrm{m}$ maps and $H S T H_{160}$-band imaging are shown in Figure 6. These growth curves in both cases were calculated using a 1". 5 radius aperture centered on the ALMA emission, assuming that this is indicating the mass-weighted center of the system. The ALMA $870 \mu \mathrm{m}$ curve dips below a cumulative fraction of 1.0 at large $(>0$." 6$)$ radii due to the presence of negative sidelobes in the ALMA maps. Calculated in this way, the median half-light radius of the ALMA $870 \mu \mathrm{m}$ emission is 0 " $16 \pm 0$ ". 02 , in agreement with the direct integration value given in Appendix A.1, while the median half-light radius of the $H_{160}$-band imaging is $0 . " 5 \pm 0$." 1 -a factor of three larger.

These comparisons also clearly demonstrate the morphological contrast between the internal structure of the obscured and unobscured star formation. While we find evidence that the obscured star formation is distributed in smooth exponential disks at a resolution of $\sim 0$ ". 16 , the stellar emission on the same scales appears very clumpy and irregular. Chen et al. (2015) studied the stellar morphologies of a larger sample of 48 ALESS SMGs (including those presented here) and reported that of the $\sim 80 \%$ detected in the $H_{160}$-band down to a median sensitivity of $H_{160}=27.8 \mathrm{mag}, 82 \pm 9 \%$ appear to have disturbed morphologies. This implies that the irregular stellar morphologies we observe are representative of the larger sample. Based on a statistical comparison with the lowerresolution Cycle 0 data, Chen et al. (2015) also reported an offset between the $H_{160}$-band components and the dusty starforming regions, which they argued could be due to either obscuration of the rest-frame optical/UV imaging or real misalignment between the dusty star-forming regions and the location of the majority of the unobscured stellar continuum emission within the SMGs. They argue that the latter scenario is more likely, given the lack of a difference between the lowand high-redshift subsamples, as the morphological $K$-correction implies that the rest-frame UV emission traced in higherredshift sources will be more sensitive to clumpy star-forming regions and dust obscuration. The present comparison demonstrates that the asymmetric, morphologically complex stellar emission indeed appears to be largely uncorrelated with 

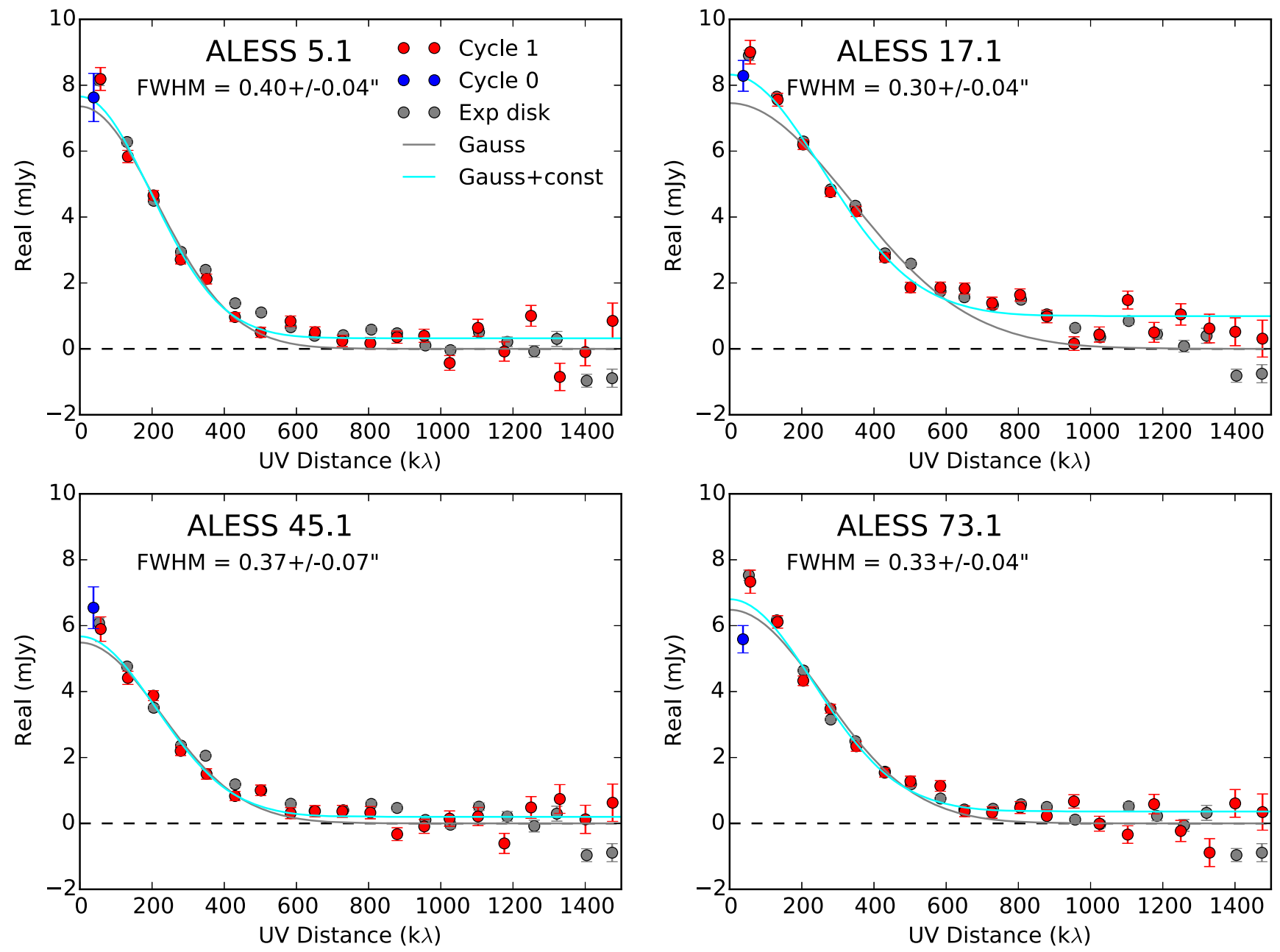

Figure 4. Visibility $(u v)$-profiles for four isolated SMGs from our study. The new observations (Cycle 1) have been phase-shifted to center on the source of interest and subsequently radially averaged in bins of $75 \mathrm{k} \lambda$ (red circles). The low-resolution Cycle 0 data had a similar procedure applied, and the results were then scaled by the response of the Cycle 0 primary beam at the source position (blue circles). Also shown are simulated profiles (with noise added) of exponential disks $(n=1)$ with the same approximate flux densities, effective radii, axial ratios, and $u v$-coverage as the sources (gray circles). Two fits to the Cycle 1 data are shown: (1) A single Gaussian fit; and (2) a Gaussian plus constant, where the latter corresponds to a point source (or point sources) in the image plane. The FWHM listed is from the second fit. We find no evidence that our new Cycle 1 data are resolving out extended emission, in agreement with Section 2.3, and we conclude that the sizes measured in the image plane are robust.

the sites of the ongoing dusty star formation on a case-by-case basis, confirming that the misalignment is real.

We conclude that the obscured star formation traced by the ALMA $870 \mu \mathrm{m}$ emission is both significantly smoother and more compact than the unobscured stellar emission. However, it is possible that the resolution of the current ALMA data ( $\sim 0$ ". 16; $\sim 1.3 \mathrm{kpc}$ at $z \sim 2.5$ ) is still slightly too coarse to resolve any potential clump-like structure. We investigate whether the dust emission shows evidence for clumpy structure as we push down to smaller spatial scales in the next section.

\subsection{Clumps}

Massive $\left(\sim 10^{8}-10^{10} M_{\odot}\right) \quad$ kiloparsec-scale star-forming clumps have been argued to be an important feature of highredshift galaxies, with observational evidence claimed for such clumps in the rest-frame UV (e.g., Elmegreen \& Elmegreen 2005; Guo et al. 2012), rest-frame optical (e.g., Elmegreen et al. 2009; Förster Schreiber et al. 2011), NIR integral field spectroscopy (e.g., Genzel et al. 2008, 2011), and potentially also $\mathrm{CO}$ and rest-frame FIR emission in a handful of the brightest and/or strongly lensed sources (e.g., Swinbank et al. 2010b, 2011; Tacconi et al. 2010; Hodge et al. 2012; Oteo et al. 2016). It has been proposed that these clumps form in-situ from the fragmentation of gravitationally unstable gas disks (e.g., Noguchi 1998; Agertz et al. 2009; Bournaud et al. 2012); though, it has also been suggested that some of the most massive clumps may be accreted cores of satellite galaxies (e.g., Mandelker et al. 2017; Oklopcic et al. 2016), and reconciling the existence of such clumps with the presence of certain stellar feedback recipes makes them an important testbed of feedback processes in galaxy formation (e.g., Mayer et al. 2016). To search for such clumps in our SMGs, we re-image the ALMA $870 \mu \mathrm{m}$ data with a Briggs robust parameter of $R=-0.5$, resulting in a resolution of $0{ }^{\prime \prime} 12 \times 0$ !" 11 . This results in almost a factor of two decrease in beam area over the "native" resolution, corresponding to physical scales of $1.0 \times 0.9 \mathrm{kpc}$ at $z \sim 2.5$. As a consequence, the typical rms noise values in the maps approximately double to $\sim 130 \mu \mathrm{Jy}_{\text {beam }}{ }^{-1}$.

Figure 7 shows several examples of SMGs imaged in this way, where we have selected those which are clumpiest in appearance. It is tempting to conclude from a visual inspection 


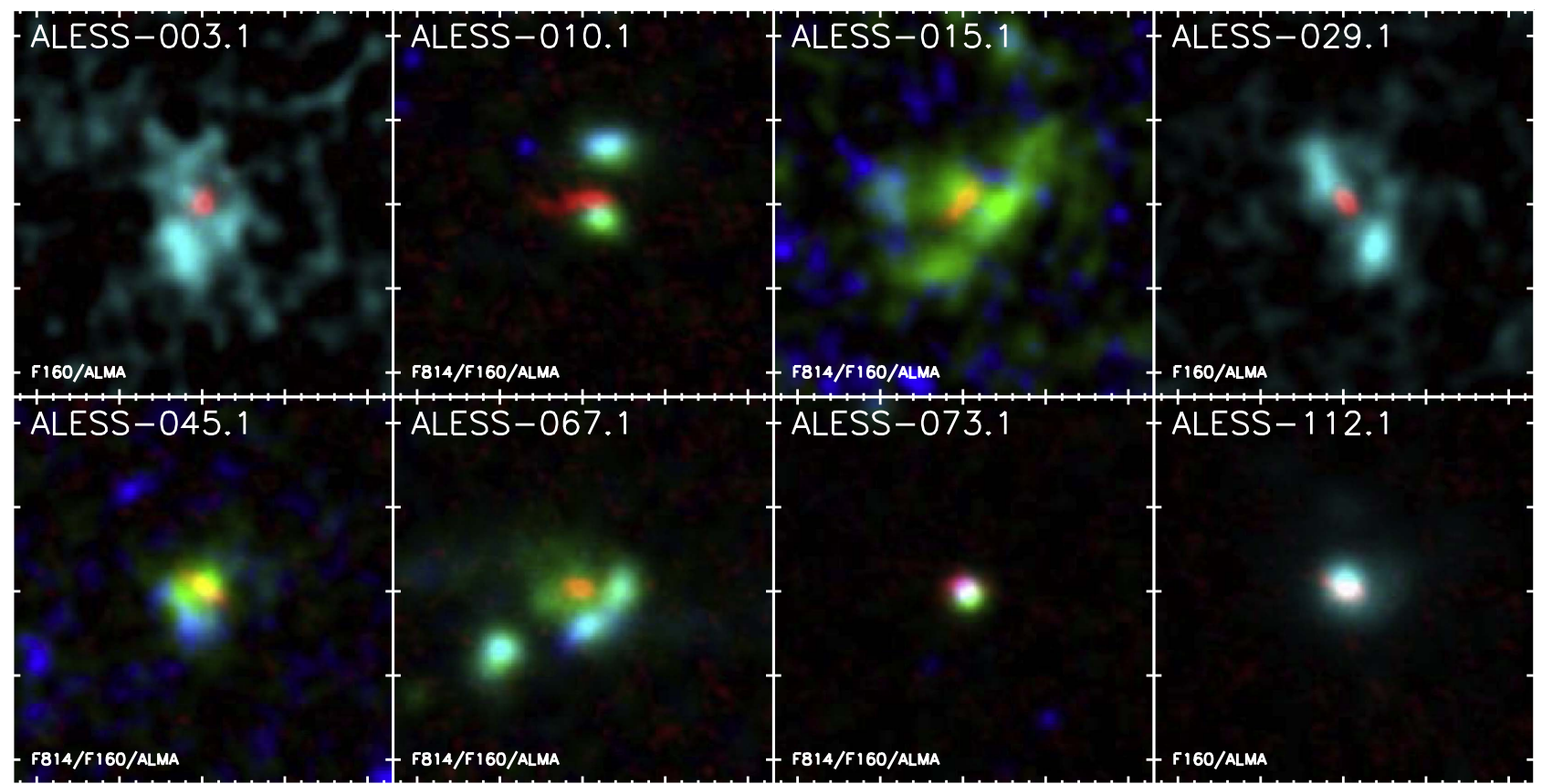

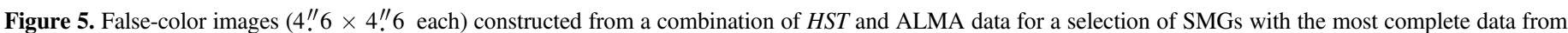

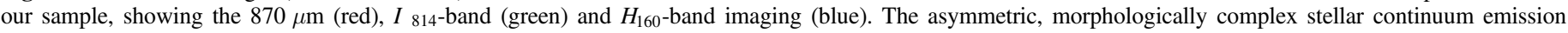
appears to be largely uncorrelated with the sites of the significantly more compact and disk-like ongoing dusty star formation

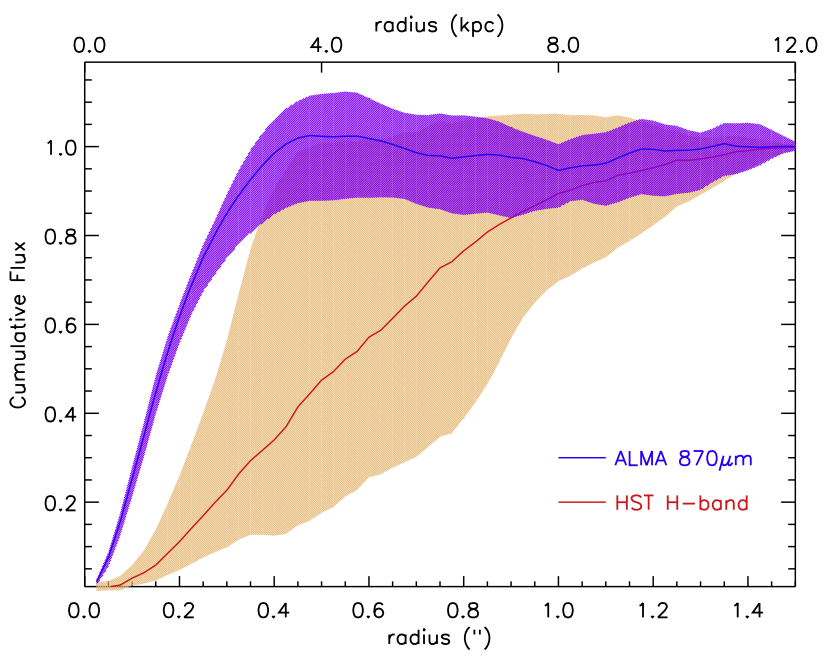

Figure 6. Curves of growth for the fraction of the flux density within a 1.5 radius aperture in the ALMA $870 \mu \mathrm{m}$ and $H S T H_{160}$-band images. The solid lines show the median, and the shaded regions show the source-to-source scatter. The top axis denotes the physical scale for a typical redshift of $z \sim 2.5$. The obscured star formation traced by the ALMA $870 \mu \mathrm{m}$ emission appears to be significantly more compact than the unobscured stellar emission.

that several of the SMGs break up into a small number of kiloparsec-scale clumps. To test this, we used CASA to simulate 16 observations of smooth exponential disks with the same angular resolution and noise levels as the observations in Figure 7. The parameters of the input model were tuned to the typical parameters observed in our SMGs: an effective radius of $R_{e}=0$." 26 , an axis ratio of 0.5 , and a total flux density of $\mathrm{S}_{870 \mu \mathrm{m}} \sim 6.5 \mathrm{mJy}$. Several examples of simulated maps are shown in Figure 7 along with the real data, where, just as with the real data, we have selected those that are clumpiest in appearance. Indeed, many of the simulated exponential disks break up into a small number of closely spaced emission peaks, similar to the observed high-resolution maps. This experiment highlights that caution should be exercised when identifying structure in high-resolution interferometric maps at this $\mathrm{S} / \mathrm{N}$ level $(\mathrm{S} / \mathrm{N} \sim 5-10)$.

As a more quantitative analysis, we fit each observed SMG with a $2 \mathrm{D}$ elliptical Gaussian and subtracted the resulting model of the smooth emission from the high-resolution map. We find that none of the SMGs have residual structures with peak fluxes $>3 \sigma$. Of the six SMGs with residuals between 2.5 and $3 \sigma$, the strongest residual $(2.9 \sigma)$ is due to the possible structure to the east of the main peak in ALESS 73.1 (Figure 7).

Recognizing that any smooth contribution may be overestimated by this crude method, we note that in all of the sources except for ALESS 15.1, the candidate clumps are only distinct from each other at the 1-2 $\sigma$ level even before the subtraction, again consistent with the smooth-disk simulations. The clump candidates in ALESS 15.1 (Figure 7) are the only candidates that are separated in brightness by $>4 \sigma$ in the highresolution maps. These candidates have peak flux densities of $0.8-1.0 \mathrm{mJy}$ beam $^{-1}$, integrated flux densities of $2.8-4.2 \mathrm{mJy}$, and FWHM areas of $2.3-2.8 \mathrm{kpc}^{2}$ (assuming $z_{\text {phot }}=1.93$; Simpson et al. 2014). If this structure is real, then scaling the total estimated star-formation rate $\left(130 M_{\odot} \mathrm{yr}^{-1}\right.$; Swinbank et al. 2014) by the ratio of the integrated flux density in each clump over that of the source as a whole gives star-formation rate surface densities of $\sim 15-20 M_{\odot} \mathrm{yr}^{-1} \mathrm{kpc}^{-2}$ (see Simpson et al. 2015b). It is possible that these two clump-like structures are the cores of merging galaxies, though we have no way to distinguish between these scenarios with the current data. We find no strong evidence for corresponding structure in the HST $H_{160}$-band image (see Figure 11 ), though the counterpart is very faint. We conclude that while there may be a hint of clump-like dust emission in the current $870 \mu \mathrm{m}$ data on $\sim$ kiloparsec scales, higher signal-to-noise observations at higher spatial resolution are required to confirm whether these clumpy structures are indeed real. 


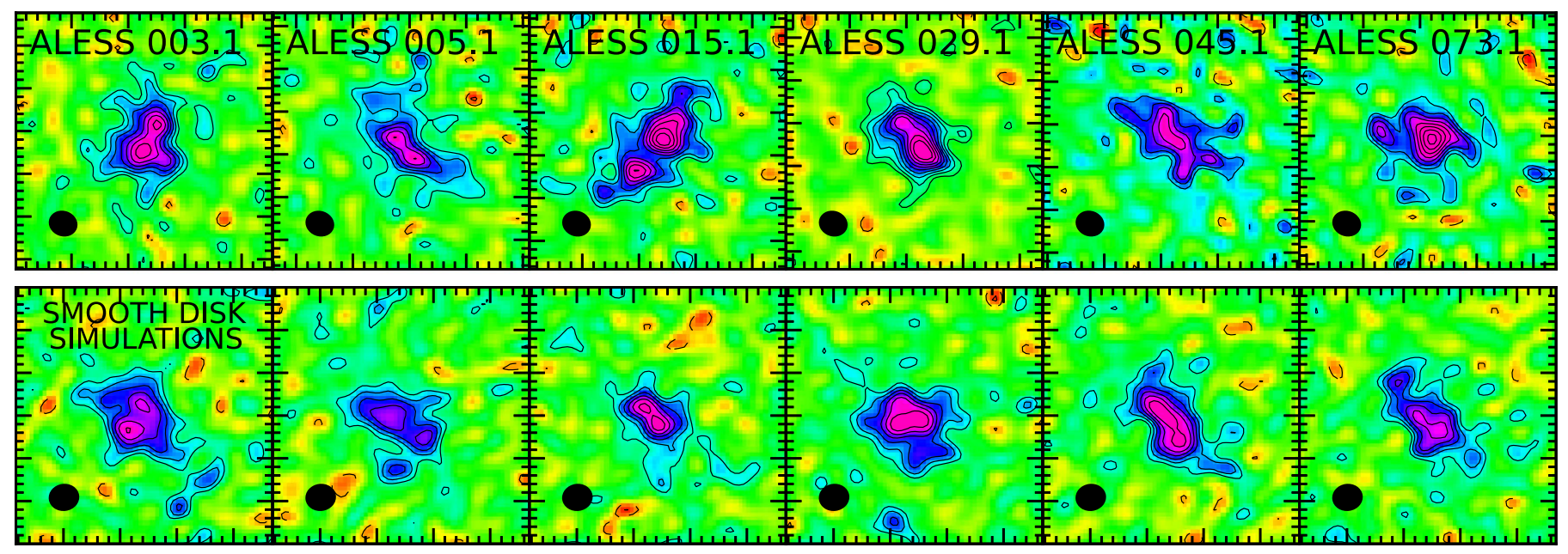

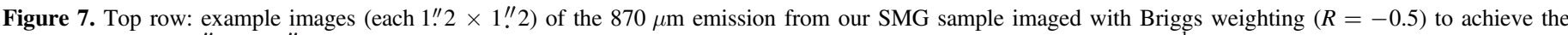

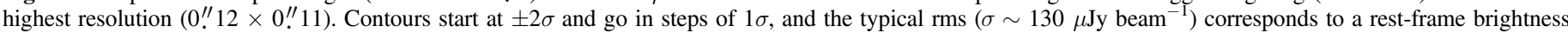

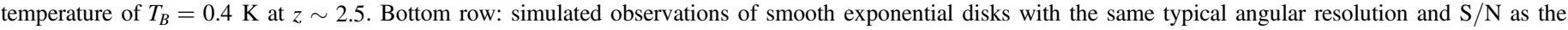

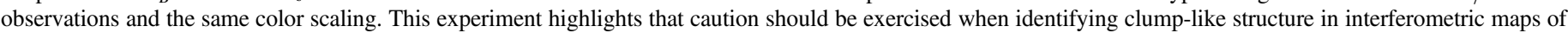
even moderate $\mathrm{S} / \mathrm{N}$.

\subsection{The $L_{\mathrm{IR}}-T$ Relation and Gas Surface Densities}

Infrared-luminous galaxies in the local (e.g., Chapin et al. 2009; Hwang et al. 2010) and high-redshift universe (e.g., Blain et al. 2003; Chapman et al. 2003a) have long been known to show a relation between their dust temperatures and infrared luminosities (the $T_{\text {dust }}-L_{\mathrm{IR}}$ relation). This relation is not due simply to selection effects, but is instead a consequence of the Stefan-Boltzmann law relating size, luminosity, and dust temperature. We plot this relation as well as the $T_{\text {dust }}-R_{\mathrm{e}}$ relation for our SMGs in Figure 8, where we have used the $L_{\text {FIR }}$ and $T_{\text {dust }}$ values reported in Swinbank et al. (2014) for those sources without updated spectroscopic redshifts (Danielson et al. 2016). The tracks plotted indicate different physical sizes of a perfect blackbody, and we assume optically thick radiation. We see that the physical scale of the dust emission correlates with redshift and dust temperature. We also see a strong $T_{\text {dust }}-L_{\mathrm{IR}}$ relation implying sizes of $1-2 \mathrm{kpc}$ (with marginal evidence that the dust emission in higher-luminosity SMGs is more compact). The sizes we measure directly from the highresolution maps (median $R_{e}=1.8 \pm 0.2 \mathrm{kpc}$ ) are in agreement with the predictions of this simple model. This result contrasts with the conclusion of Yan \& Ma (2016) based on the modified blackbody equivalent of the Stefan-Boltzmann law applied to strongly lensed sources, where they suggested that the larger sizes measured for their high-redshift sources must be the result of blending. We note that when we use the modified blackbody equivalent of the Stefan-Boltzmann law instead for our sample, the sizes we measure are still consistent with the predictions (median ratio of $R_{\text {eff,predicted }} / R_{\text {eff, observed }}=1.1 \pm 0.2$ ).

We then used the resolved nature of our observations to search for correlations between dust/gas surface density and dust temperature/luminosity. Figure 8 shows $870 \mu \mathrm{m}$ surface brightness as a function of dust temperature, where we include measurements of both the average and peak surface brightness in each galaxy. No trend is evident between the surface brightness and dust temperature. We have then converted these measurements to gas surface density by scaling the SEDderived dust mass and assuming a gas-to-dust ratio of 100. The corresponding extinction $\left(A_{\mathrm{v}}\right)$ values were calculated as in Güver \& Özel (2009). The gas surface densities implied by our observations are very high-over two orders of magnitude higher than GMCs in the nearby universe (Solomon et al. 1987) - and similar to those found in local ULIRGs. There appears to be no trend between gas surface density/extinction and total infrared luminosity.

\section{DISCUSSION}

The ALMA imaging presented here allows us to resolve the dust-obscured star formation in a sample of luminous highredshift dusty star-forming galaxies on scales of $\sim 1 \mathrm{kpc}$. Sérsic profile fits reveal that the galaxies have a median effective radius of $R_{e}=0$ ". $24 \pm 0$ ". 02 at a rest wavelength of $\lambda \sim 250 \mu \mathrm{m}$ (for a typical source redshift of $z \sim 2.5$ ), corresponding to a typical physical size of $R_{e}=1.8 \pm$ $0.2 \mathrm{kpc}$. In contrast, Herschel 70-160 $\mu \mathrm{m}$ imaging of 400 local galaxies and QSO hosts suggests that ULIRGs are exclusively found with very compact $\left(R_{e} \sim 0.5 \mathrm{kpc}\right)$ morphologies (albeit at shorter rest wavelengths of $\lambda \sim 70 \mu \mathrm{m}$; Lutz et al. 2016). This confirms earlier suggestions from $\mathrm{CO}$ observations and marginally resolved radio and submillimeter data (e.g., Chapman et al. 2004; Carilli et al. 2010; Ivison et al. 2010, 2011; Hodge et al. 2013a; De Breuck et al. 2014; Gilli et al. 2014; Simpson et al. 2015b; Ikarashi et al. 2015; Miettinen et al. 2015) that high-redshift dusty star-forming galaxies are indeed larger than similarly luminous local galaxies.

In addition to the observed sizes, the observations presented here resolve the dust emission over many beams at relatively high $\mathrm{S} / \mathrm{N}$, allowing us to constrain the more detailed morphology. In particular, there have been a number of claims in the literature that, when observed at high-resolution, the gas reservoirs of SMGs break up into sub-kiloparsec or kiloparsecsized clumps (e.g., Tacconi et al. 2010; Swinbank et al. 2011; Hodge et al. 2012; Hatsukade et al. 2015). Assuming a constant dust-to-gas ratio-i.e., that the dust follows the gas - the dust distribution should then be similarly clumpy. Such clumpy dust within a rotating gas disk was potentially observed in, for example, the strongly lensed "Eyelash" galaxy by Swinbank et al. (2010b), seeming to confirm this theory. In contrast, we find that the SMGs observed here appear (within the limits of our current resolution and sensitivity) to be smooth and disk- 

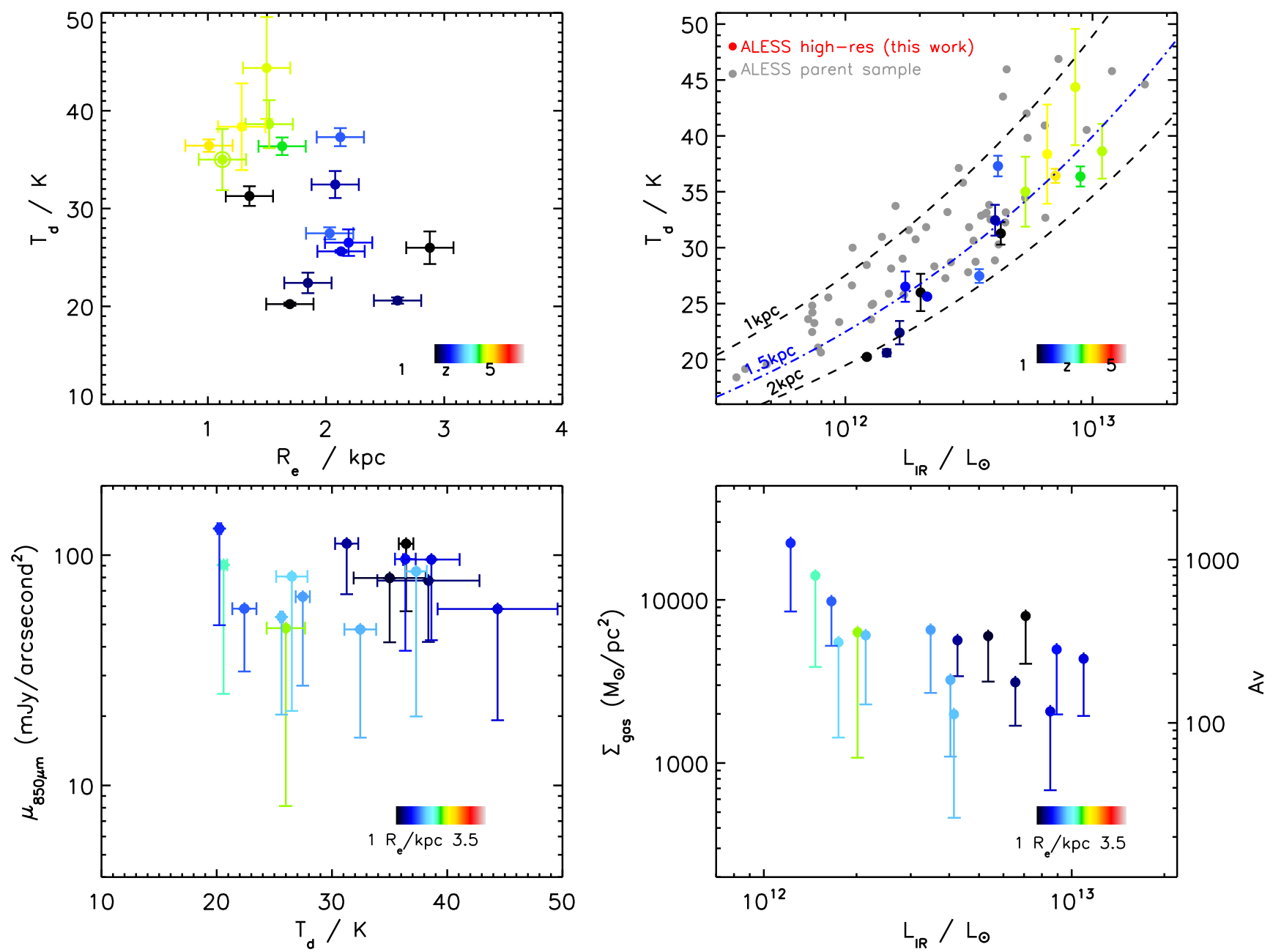

Figure 8. Top left: the characteristic dust temperature $\left(T_{\mathrm{d}}\right)$ from a modified blackbody fit (Swinbank et al. 2014) vs. effective radius $\left(R_{\mathrm{e}}\right)$, color coded by source redshift. The dust emission in the higher-redshift sources appears to be warmer and more compact. Top right: the characteristic dust temperature vs. infrared luminosity $\left(L_{\mathrm{IR}}\right)$ for the targets of this paper compared to the parent ALESS sample. The high-resolution sources (this work) are again color coded by redshift. The dashed lines indicate the physical sizes predicted assuming the Stefan-Boltzmann law. The sizes we derive are consistent with those predicted from this simple model, and there is marginal evidence that the SMGs with the highest luminosities are more compact. Bottom left: the peak (filled) and average (lower error bar) $870 \mu \mathrm{m}$ surface brightness from the highest-resolution maps (Section 3.3) vs. characteristic dust temperature, color coded by physical size. There is no trend in surface brightness with dust temperature. Bottom right: the gas surface density vs. infrared luminosity, color coded by physical size. The right-hand axis shows the corresponding visual extinction $\left(A_{\mathrm{v}}\right)$. The gas surface density/extinction values measured are very high, but there appears to be no trend with infrared luminosity.

like on kiloparsec scales, with a median Sérsic index of $n=0.9 \pm 0.2$. Combined with the measured sizes $\left(R_{e}=1.8 \pm 0.2 \mathrm{kpc}\right)$, this seems to rule out the sort of extended, clumpy disk galaxies predicted by simulations of violent disk instability (e.g., Dekel et al. 2009; Bournaud et al. 2014) and observed in optically bright systems (e.g., Förster Schreiber et al. 2006) and potentially even in the ultraluminous $z \sim 4$ SMG GN20 (Hodge et al. 2012, 2015). The relative uniformity of the dust morphologies observed here also seems to contradict models where SMGs are a heterogenous population (e.g., Hayward et al. 2011, 2012); though, larger sample sizes covering a larger range of flux densities are required to more thoroughly test this conclusion.

It is, of course, still possible that there is a clump-like structure below our current resolution limits. The clumps in the Eyelash and SDP.81 are reported to have physical sizes of only a couple hundred parsecs (Swinbank et al. 2010b; Hatsukade et al. 2015). Similarly, the dust continuum in the most wellstudied local ULIRG, Arp 220, is concentrated in two very compact $(\sim 30-50 \mathrm{pc})$ nuclei situated $\sim 300 \mathrm{pc}$ apart (although at longer rest-frame wavelengths; e.g., Sakamoto et al. 2008; Barcos-Muñoz et al. 2015; Scoville et al. 2016). We would not be able to resolve the nuclei of Arp 220 at a redshift of $z \sim 2.5$ with the present observations, and indeed, we may find a hint of clump-like structure in one of our SMGs when we push down to (sub-)kiloparsec scales. However, the simulations and analysis in Section 3.3 suggest that caution should be exercised when identifying candidate clumps in even moderate $\mathrm{S} / \mathrm{N}$ interferometric data. Indeed, the sizes we measure from the high-resolution images are consistent with those predicted from the Stefan-Boltzmann law based on the measured dust temperatures and FIR luminosities, another indication that the emission is relatively smooth. The measured sizes also agree with those estimated from fitting models assuming power-law mass-temperature distributions, again assuming smooth-disk emission (Kovács et al. 2010). Significantly higher-S/N observations at higher resolution are required to determine whether the dust emission in these SMGs retains a disk-like appearance on sub-kiloparsec scales. 
In contrast to the smooth appearance of the obscured star formation, the matched-resolution HST WFC3 imaging of these SMGs-tracing the unobscured rest-frame optical lightappears clumpy and irregular. The median half-light radius observed for the unobscured stellar emission in these sources corresponds to $R_{e}=4.1 \pm 0.8 \mathrm{kpc}$ at $z \sim 2.5$, implying that the pre-existing stellar distributions of the SMGs are also significantly more extended than the dust emission. A similar conclusion was drawn regarding the morphology and extent of the stellar component for the larger sample of 48 ALESS SMGs presented by Chen et al. (2015), indicating that stellar morphologies observed in our sources are representative of the parent population. The current study reveals that this unobscured stellar emission is largely uncorrelated with the obscured star-forming regions in individual sources. This observation implies that SED fitting routines assuming a simple dust screen over a single or even composite stellar population may be too simplistic.

The difference observed between the morphology of the obscured star formation and unobscured stellar emission in these SMGs also leads us to consider their formation scenario. Chen et al. (2015) use the apparently disturbed rest-frame optical morphologies, along with the short expected lifetimes of SMGs, to argue that the majority of $z \sim 2-3$ SMGs are early/mid-stage major mergers, as has been argued previously on the basis of, e.g., radio and submillimeter multiplicity and kinematics (e.g., Swinbank et al. 2006; Engel et al. 2010). Theoretically, the profiles of merger remnants are expected to be relatively compact and strongly centrally peaked due to the violent and dissipative collapse expected in turbulent and clumpy gas (e.g., Bournaud et al. 2011). The small sizes of the dust disks we measure could be consistent with this scenario, though the observed Sérsic indices are lower than expected in the simulations.

If the starbursts in these galaxies are major merger driven, we are likely observing the result of the gas/dust more rapidly (re-)forming disk structures than the existing stellar component. Assuming a typical gas consumption timescale for SMGs of $\sim 100$ Myr (Bothwell et al. 2013), and based on the apparent dynamical (orbital) timescales ( $20 \mathrm{Myr})$ implied assuming velocity widths of $\sim 500 \mathrm{~km} \mathrm{~s}^{-1}$ (Bothwell et al. 2013) and the effective radii measured here, it is possible that the disks have settled while the burst of star formation is still ongoing. It is possible that the more compact stellar counterparts observed in some sources (Figure 5) then correspond to more evolved systems. Simulations show that the old stars present in the existing stellar component may also contract due to the turbulent dissipation of the gas and young stars, which can contain a large fraction of the total mass (Bournaud et al. 2011). The current bursts of star formation thus have the potential to transform both the observed galaxy sizes and the overall light profiles as they evolve.

This transformation could also help establish the connection between SMGs and local elliptical galaxies, their proposed descendants (e.g., Eales et al. 1999; Swinbank et al. 2006; Ikarashi et al. 2015). In Figure 9, we compare the properties of the ALESS SMGs studied in this work with the volume-limited ATLAS $^{3 \mathrm{D}}$ sample of nearby early-type galaxies (Cappellari et al. 2011). The stellar masses, effective radii, and mass surface densities for the ATLAS ${ }^{3 \mathrm{D}}$ galaxies are discussed in Cappellari et al. (2013). The median properties ${ }^{21}$ of the ALESS

\footnotetext{
${ }^{21}$ We show the median properties of the ALESS SMGs because there can be significant scatter among individual galaxies.
}

SMGs from this work are overplotted, where we use the average gas mass surface densities (Figure 8). If we assume an average stellar mass of $M_{*} \sim 8 \times 10^{11} M_{\odot}$ (Simpson et al. 2014) and a gas mass of $M_{\mathrm{gas}} \sim 5 \times 10^{10} M_{\odot}$ (Bothwell et al. 2013; consistent with that derived from the dust masses for our sources), then the $z \sim 0$ descendants of these SMGs would have total masses of $M_{*} \sim 1-2 \times 10^{10} M_{\odot}$ (assuming $\sim 100 \%$ star formation efficiency in the disk). If we then assume $z \sim 0$ sizes of $R_{\mathrm{e}} \sim 2-3 \mathrm{kpc}$ (taking the weighted average of the submillimeter and optical sizes, and assuming the stellar components may also contract further; Bournaud et al. 2011), we can estimate how the descendants of SMGs may compare to local early-type galaxies. We find that the SMG descendants have stellar masses, effective radii, and average gas surface densities consistent with the most compact massive $\left(M_{*} \sim 1-2 \times 10^{11} M_{\odot}\right)$ early-type galaxies-with the highest $M / L$ ratios-observed locally (Figure 9 ).

\section{SUMMARY}

We have presented high-resolution $(\sim 0$ !' $16 ; \sim 1.3 \mathrm{kpc}$ at $z \sim 2.5) 870 \mu \mathrm{m}$ ALMA imaging of 16 luminous ALESS SMGs, allowing us to clearly resolve the dust-obscured star formation in these $z \sim 2.5$ galaxies on $\sim 1 \mathrm{kpc}$ scales. The median light profile has an effective radius of $R_{e}=0$ "' $24 \pm 0$ " 02 (corresponding to a typical physical size of $R_{e}=1.8 \pm 0.2 \mathrm{kpc}$ ) and a Sérsic index of $n=0.9 \pm 0.2$, implying that the dust emission and, by implication, the obscured star formation is remarkably disk-like at the current resolution and sensitivity. We present a series of tests in the image and $u v$-planes to confirm that the fraction $(\sim 10 \%-15 \%)$ of emission that may be potentially "missing" from the naturally weighted maps does not bias our conclusions regarding the light profiles or sizes. Our results confirm earlier suggestions that high-redshift dusty star-forming galaxies are indeed larger than similarly luminous local galaxies.

We find that the present observations paint a different picture to that of the disturbed morphologies observed in the stellar distributions of the SMGs traced by $H S T H_{160}$-band imaging. In particular, the extended, morphologically complex stellar emission appears to be largely uncorrelated with the sites of the ongoing dusty star formation. This observation has implications for SED fitting routines assuming a simple dust screen over a single composite stellar population.

To search for clump-like structure in the dust-obscured star formation, we use different weighting schemes with the visibilities to probe scales of 0 !' $12(1.0 \mathrm{kpc})$, but we find no significant evidence for clumping in the majority of cases. Indeed, we demonstrate that the observed morphologies are generally consistent with those seen in simulated interferometric images of smooth exponential disks at similar (moderate) $\mathrm{S} / \mathrm{N}$. This experiment highlights that caution should be exercised when identifying structure in high-resolution interferometric maps at this $\mathrm{S} / \mathrm{N}$ level $(\mathrm{S} / \mathrm{N} \sim 5-10)$. While the present observations suggest that kiloparsec-scale clumps of dust (and cool gas) are rare in these systems, higher-S/ $\mathrm{N}$ observations of the dust-obscured star formation and molecular gas at higher resolution will be crucial in order to test whether the apparently smooth dust (and by implication, gas) distribution becomes more structured on sub-kiloparsec scales.

We examine a number of correlations between physical parameters for these SMGs, including the well-known $T_{\text {dust }}-L_{\mathrm{IR}}$ relation, and we find that the source sizes we measure directly 

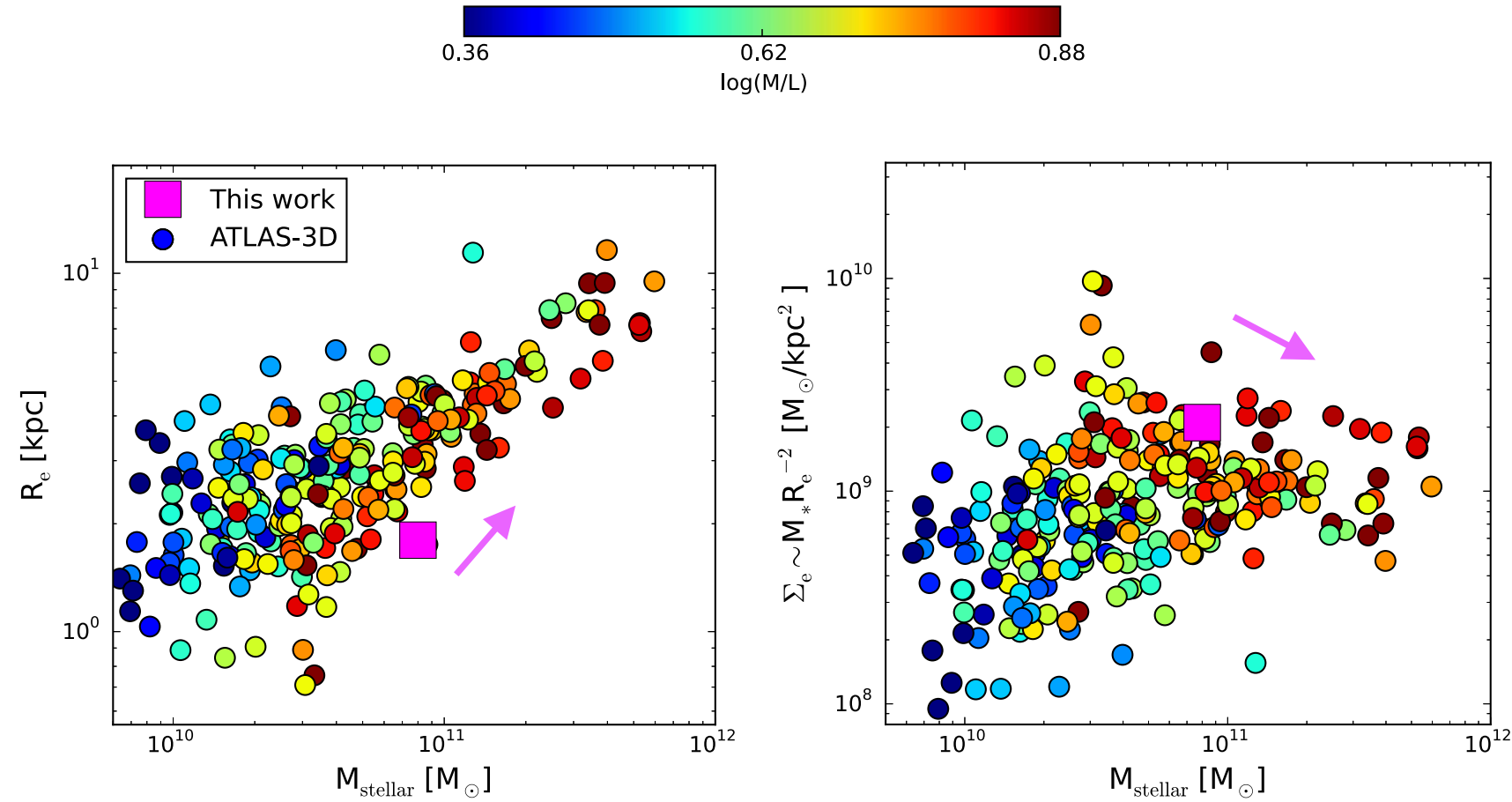

Figure 9. Comparison of the ALESS SMG properties to nearby early-type galaxies from the ATLAS ${ }^{3 \mathrm{D}}$ project. The ATLAS ${ }^{3 \mathrm{D}}$ data (circles color coded by mass-tolight ratio) come from Cappellari et al. (2011, 2013). The typical properties of the ALESS SMGs are shown, and the arrows indicate the direction that these properties may evolve in with decreasing redshift. The ALESS SMGs studied in this work have stellar masses, effective radii, and average gas surface densities similar to the locus of nearby early-type galaxies, and their descendants are thus expected to have properties similar to the most compact massive early-type galaxies observed locally.

from the high-resolution maps are consistent with those predicted by this simple relation. This agreement is another indication that the emission is relatively smooth. While the physical scale of the dust emission appears to correlate with dust temperature and redshift, no trend is evident between the surface brightness and dust temperature, nor between gas surface density/extinction and total infrared luminosity. The gas surface densities implied by our observations are significantly higher than GMCs in the nearby universe, and similar to those found in local ULIRGs.

The lack of clumps in the obscured star formation, in combination with the compact sizes, seems to rule out the sort of extended, clumpy disk galaxies predicted by simulations of violent disk instability (e.g., Dekel et al. 2009; Bournaud et al. 2014). The compact nature of the obscured star formation compared to the existing stellar component may instead suggest that the bursts are fueled by major mergers, although the exponential light profiles we observe are seemingly inconsistent with the spheroids that are thought to result from the highly dissipative collapse. The relative uniformity in the observed dust morphologies may contradict suggestions of a heterogeneous SMG population, although larger samples of galaxies covering a wider range of flux densities are required to thoroughly test this conclusion as these models suggest that the observed morphology is a function of SMG flux density.

Given the stark contrast between the observed dust and stellar morphologies, we suggest that the current bursts of star formation have the potential to transform both the observed galaxy sizes and the overall light profiles as they evolve. This transformation could help establish the connection between high-redshift SMGs and red-and-dead local elliptical galaxies, their proposed descendants. We compare the observed properties of our SMGs to the volumelimited sample of ATLAS ${ }^{3 \mathrm{D}}$ nearby early-type galaxies, and we suggest that the likely $z \sim 0$ descendants of SMGs have average properties-including stellar masses, effective radii, and gas surface densities - that are consistent with the most compact massive $\left(M_{*} \sim 1-2 \times 10^{11} M_{\odot}\right)$ early-type galaxies observed locally.

The authors wish to thank both the NAASC and the Allegro ARC node, including Todd Hunter, Alison Peck, and Michiel Hogerheijde, for assistance with collecting and analyzing the ALMA data. We also thank Romeel Davé, Marijn Franx, and Nick Scoville for useful discussions and the referee for a positive report. This work was performed in part at the Aspen Center for Physics, which is supported by National Science Foundation grant PHY-1066293. I.R.S. acknowledges support from STFC (ST/L00075/1), the ERC Advanced Grant DUSTYGAL (321334) and a Royal Society/Wolfsom Merit Award. R.J.I. acknowledges support from the European Research Council in the form of the Advanced Investigator Program, 321302, COSMICISM. F.W. acknowledges ERC Starting Grant Cosmic_Dawn. This paper makes use of the following ALMA data: ADS/JAO.ALMA\#2011.1.00294.S and ADS/JAO.ALMA\#2012.1.00307.S. A.K. acknowledges support by the Collaborative Research Council 956, sub-project A1, funded by the Deutsche Forschungsgemeinschaft (DFG). K.K. acknowledges support from the Swedish Research Council. This work was performed at the Aspen Center for Physics, which is supported by National Science Foundation grant PHY-1066293. ALMA is a partnership of ESO 

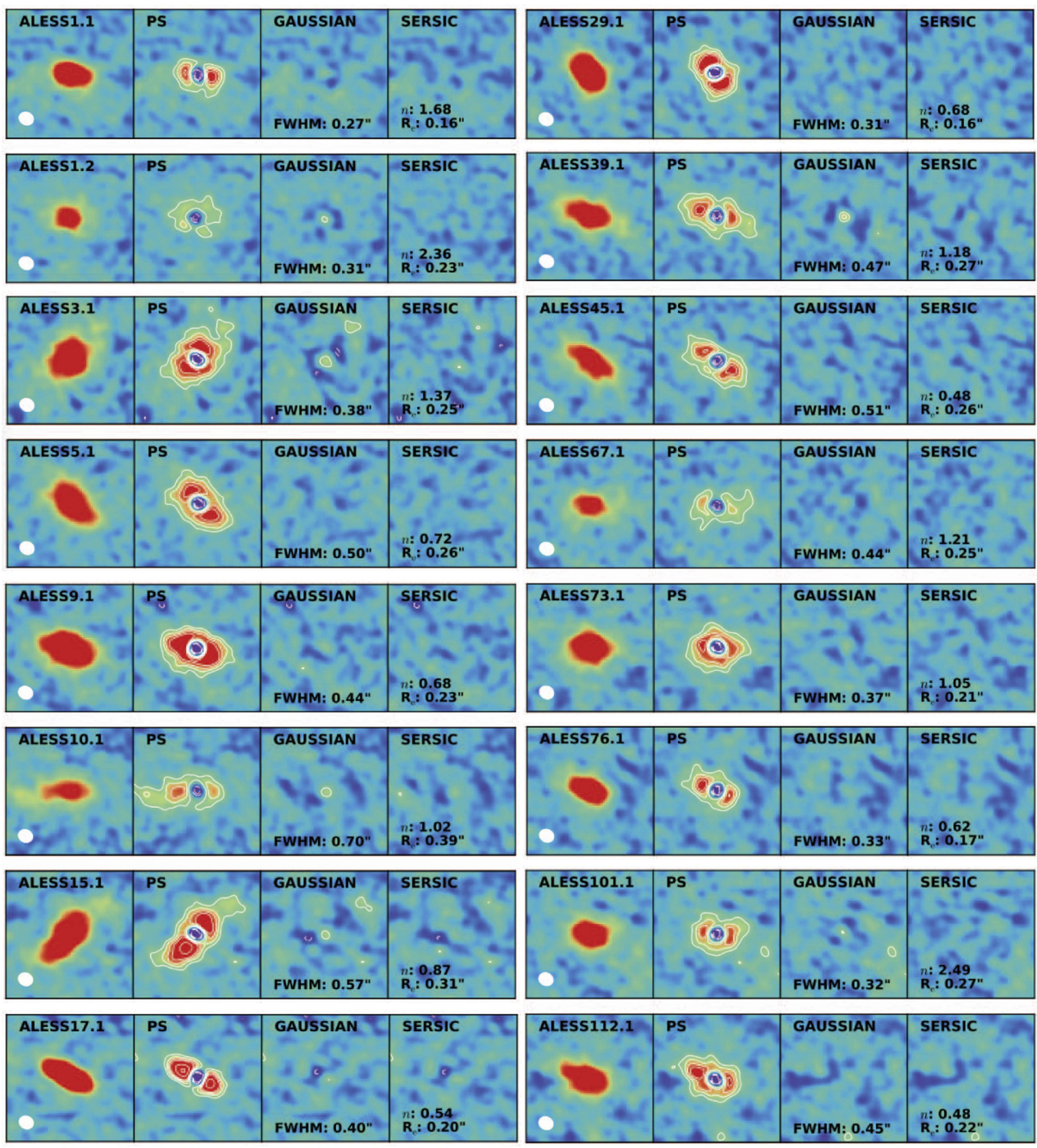

Figure 10. Images $(1$ !" $5 \times 1$ !" 5 each) showing the image-plane fitting of the emission profiles observed in the high-resolution ALMA data, including the naturally weighted images (left panel; 0 " $17 \times 0$ " 15 resolution) as well as the residuals from fitting each source with a point source (PS), two-dimensional Gaussian, and Sérsic profile. Contours in the residual panels indicate $\pm 3,5,7 \sigma$. The point source fit is ruled out by $>5 \sigma$ residuals in all cases, and we see no strong preference between Gaussian and Sérsic models.

(representing its member states), NSF (USA), and NINS (Japan), together with NRC (Canada) and NSC and ASIAA (Taiwan) and KASI (Republic of Korea), in cooperation with the Republic of Chile. The Joint ALMA Observatory is operated by ESO, AUI/NRAO, and NAOJ. The National Radio Astronomy Observatory is a facility of the National Science Foundation operated under cooperative agreement by Associated Universities, Inc. 


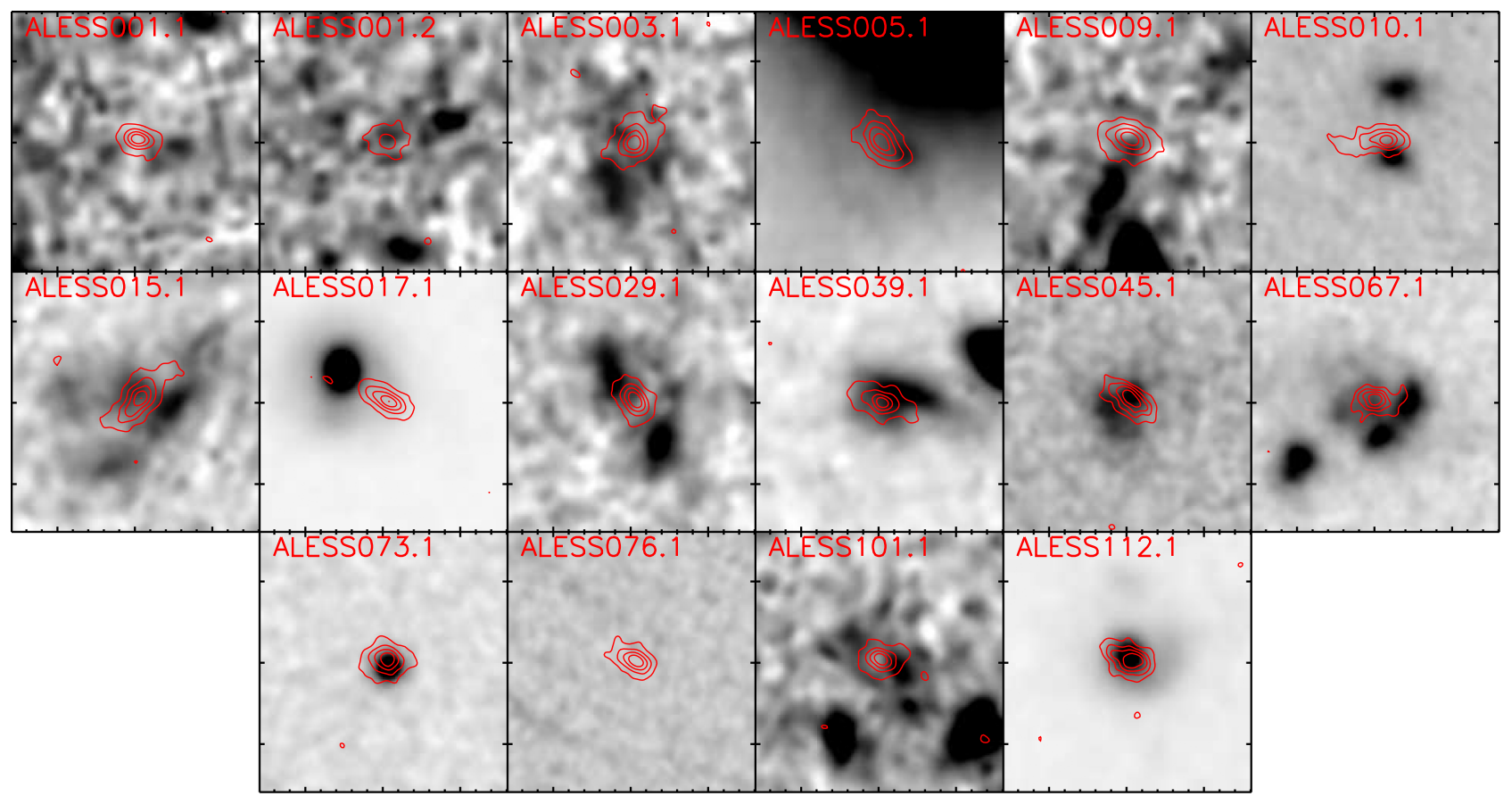

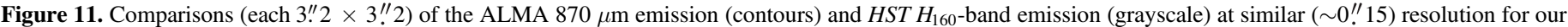

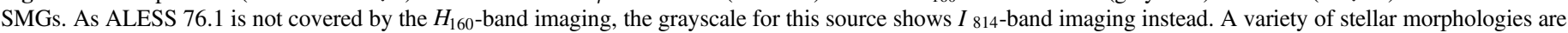
observed, and the stellar environments suggest that ALESS 5.1 and 10.1 are potentially weakly lensed by nearby foreground bright galaxies (see Section 3.2).

\section{APPENDIX}

\section{A.1. Robustness of Parameters}

While the analysis in Section 2.3 shows that the lowresolution Cycle 0 estimated flux density for each SMG is recovered in the new data, the difference between the $u v$ tapered and naturally weighted (0!"16 FWHM synthesized beam) images of the new data implies that the latter may be insensitive to a fraction $(\sim 10 \%-15 \%)$ of the emission from what is presumably a more extended component. In order to test whether this is affecting the parameters derived in the previous section, we have carried out a series of tests in the image plane.

As the first test, we fit two-dimensional Gaussian and Sérsic profiles to the data $u v$-tapered to $0 . \prime 3$. This tapering should recover the flux potentially "missing" from the naturally weighted maps (see Section 2.3) but present on the shortest baselines without degrading the image quality more than necessary. The Gaussian fits have a median major axis size of FWHM $=0$." $42= \pm 0$." 04 , and the Sérsic profile fits have a median index of $n=0.9 \pm 0.3$ and an effective radius of $R_{e}=0$ ". $21 \pm 0$ " 05 . These values are all consistent with the profiles derived from the naturally weighted maps.

As a second test, we computed the half-light radii for the sources in the naturally weighted maps by simply determining the radius within which half of the light is contained-i.e., with no preference for a particular profile. We then repeated this exercise using the total flux estimates from the $u v$-tapered maps in the denominator, and conservatively assuming that this flux lies entirely outside of the measured radii. The median half-light radii determined in this manner are $R_{e}=0$ ". $18 \pm 0$ ". 02 and $R_{e}=0$ " $19 \pm 0$ ". 02 , respectivelyshowing excellent agreement.
As a third test, we took the naturally weighted images and added $15 \%$ of the emission in a $1^{\prime \prime}$-diameter uniform disk around each source. We then re-fit the images with twodimensional Gaussian and Sérsic profiles. The resulting Gaussian fits have a median major axis size of FWHM $=0$ ". $45 \pm 0$ ". 03 , and the Sérsic profile fits have a median index of $n=1.0 \pm 0.3$ and an effective radius of $R_{e}=0$ ". $26 \pm 0$ ". 01 . These results are again consistent with the values measured from the naturally weighted maps, indicating no significant bias in the measured properties as a result of any flux potentially "missing" from the images up to the maximum estimated fraction of $10 \%-15 \%$.

\section{REFERENCES}

Agertz, O., Teyssier, R., \& Moore, B. 2009, MNRAS, 397, L64 Aravena, M., Hodge, J. A., Wagg, J., et al. 2014, MNRAS, 442, 558 Barcos-Muñoz, L., Leroy, A. K., Evans, A. S., et al. 2015, ApJ, 799, 10 Barro, G., Kriek, M., Pérez-González, P. G., et al. 2016, ApJL, 827, L32 Blain, A. W., Barnard, V. E., \& Chapman, S. C. 2003, MNRAS, 338, 733 Blain, A. W., Smail, I., Ivison, R. J., et al. 2002, PhR, 369, 111 Bolatto, A. D., Warren, S. R., Leroy, A. K., et al. 2015, ApJ, 809, 175 Bothwell, M. S., Smail, I., Chapman, S. C., et al. 2013, MNRAS, 429, 3047 Bothwell, M. S., Chapman, S. C., Tacconi, L., et al. 2010, MNRAS, 405, 219 Bournaud, F. 2016, Galactic Bulges, 418, 355

Bournaud, F., Chapon, D., Teyssier, R., et al. 2011, ApJ, 730, 4 Bournaud, F., Juneau, S., Le Floc'h, E., et al. 2012, ApJ, 757, 81 Bournaud, F., Perret, V., Renaud, F., et al. 2014, ApJ, 780, 57 Cappellari, M., Emsellem, E., Krajnović, D., et al. 2011, MNRAS, 413, 813 Cappellari, M., McDermid, R. M., Alatalo, K., et al. 2013, MNRAS, 432, 1862 Carilli, C. L., Daddi, E., Riechers, D., et al. 2010, ApJ, 714, 1407 Carilli, C. L., \& Walter, F. 2013, ARA\&A, 51, 105

Casey, C. M., Narayanan, D., \& Cooray, A. 2014, PhR, 541, 45 Chapin, E. L., Hughes, D. H., \& Aretxaga, I. 2009, MNRAS, 393, 653

Chapman, S. C., Blain, A. W., Smail, I., \& Ivison, R. J. 2005, ApJ, 622, 772 Chapman, S. C., Helou, G., Lewis, G. F., \& Dale, D. A. 2003a, ApJ, 588, 186 Chapman, S. C., Smail, I., Windhorst, R., et al. 2004, ApJ, 611, 732

Chapman, S. C., Windhorst, R., Odewahn, S., Yan, H., \& Conselice, C. 2003b, ApJ, 599, 92

Chen, C.-C., Smail, I., Swinbank, A. M., et al. 2015, ApJ, 799, 194 
Cornwell, T. J. 2008, ISTSP, 2, 793

Daddi, E., Alexander, D. M., Dickinson, M., et al. 2007a, ApJ, 670, 173

Daddi, E., Bournaud, F., Walter, F., et al. 2010, ApJ, 713, 686

Daddi, E., Cimatti, A., Renzini, A., et al. 2004, ApJ, 617, 746

Daddi, E., Dickinson, M., Morrison, G., et al. 2007b, ApJ, 670, 156

Danielson, A. L. R., Swinbank, A. M., Smail, I., et al. 2017, ApJ, submitted

Dannerbauer, H., Walter, F., \& Morrison, G. 2008, ApJL, 673, L127

Davé, R., Finlator, K., Oppenheimer, B. D., et al. 2010, MNRAS, 404, 1355

De Breuck, C., Williams, R. J., Swinbank, M., et al. 2014, A\&A, 565, A59

Dekel, A., Sari, R., \& Ceverino, D. 2009, ApJ, 703, 785

Dekel, A., Zolotov, A., Tweed, D., et al. 2013, MNRAS, 435, 999

Eales, S., Lilly, S., Gear, W., et al. 1999, ApJ, 515, 518

Elmegreen, B. G., Bournaud, F., \& Elmegreen, D. M. 2008, ApJ, 688, 67

Elmegreen, B. G., \& Elmegreen, D. M. 2005, ApJ, 627, 632

Elmegreen, B. G., Elmegreen, D. M., Fernandez, M. X., \& Lemonias, J. J. 2009, ApJ, 692, 12

Elmegreen, D. M., Elmegreen, B. G., \& Sheets, C. M. 2004, ApJ, 603, 74

Engel, H., Tacconi, L. J., Davies, R. I., et al. 2010, ApJ, 724, 233

Förster Schreiber, N. M., Shapley, A. E., Genzel, R., et al. 2011, ApJ, 739, 45

Förster Schreiber, N. M., Genzel, R., Lehnert, M. D., et al. 2006, ApJ, 645,1062

Genzel, R., Newman, S., Jones, T., et al. 2011, ApJ, 733, 101

Genzel, R., Tacconi, L. J., Gracia-Carpio, J., et al. 2010, MNRAS, 407, 2091

Genzel, R., Tacconi, L. J., Kurk, J., et al. 2013, ApJ, 773, 68

Genzel, R., Burkert, A., Bouche, N., et al. 2008, ApJ, 687, 59

Gilli, R., Norman, C., Vignali, C., et al. 2014, A\&A, 562, A67

Guo, Y., Ferguson, H. C., Bell, E. F., et al. 2015, ApJ, 800, 39

Guo, Y., Giavalisco, M., Ferguson, H. C., Cassata, P., \& Koekemoer, A. M. 2012, ApJ, 757, 120

Güver, T., \& Özel, F. 2009, MNRAS, 400, 2050

Hatsukade, B., Tamura, Y., Iono, D., et al. 2015, PASJ, 67, 93

Hayward, C. C., Jonsson, P., Kereš, D., et al. 2012, MNRAS, 424, 951

Hayward, C. C., Kereš, D., Jonsson, P., et al. 2011, ApJ, 743, 159

Hodge, J. A., Carilli, C. L., Walter, F., et al. 2012, ApJ, 760, 11

Hodge, J. A., Carilli, C. L., Walter, F., et al. 2013a, ApJ, 776, 22

Hodge, J. A., Karim, A., Smail, I., et al. 2013b, ApJ, 768, 91

Hodge, J. A., Riechers, D., Decarli, R., et al. 2015, ApJL, 798, L18

Hwang, H. S., Elbaz, D., Magdis, G., et al. 2010, MNRAS, 409, 75

Ikarashi, S., Ivison, R. J., Caputi, K. I., et al. 2015, ApJ, 810, 133

Ivison, R. J., Papadopoulos, P. P., Smail, I., et al. 2011, MNRAS, 412, 1913

Ivison, R. J., Smail, I., Papadopoulos, P. P., et al. 2010, MNRAS, 404, 198

Karim, A., Swinbank, A. M., Hodge, J. A., et al. 2013, MNRAS, 432, 2

Kereš, D., Katz, N., Davé, R., Fardal, M., \& Weinberg, D. H. 2009a, MNRAS, 396,2332
Kereš, D., Katz, N., Fardal, M., Davé, R., \& Weinberg, D. H. 2009b, MNRAS, 395, 160

Kereš, D., Katz, N., Weinberg, D. H., \& Davé, R. 2005, MNRAS, 363, 2

Kovács, A., Omont, A., Beelen, A., et al. 2010, ApJ, 717, 29

Lutz, D., Berta, S., Contursi, A., et al. 2016, A\&A, 591, A136

Mandelker, N., Dekel, A., Ceverino, D., et al. 2017, MNRAS, 464, 635

Mayer, L., Tamburello, V., Lupi, A., et al. 2016, ApJ, 830, 13

Miettinen, O., Novak, M., Smolčić, V., et al. 2015, A\&A, 584, A32

Narayanan, D., Hayward, C. C., Cox, T. J., et al. 2010, MNRAS, 401, 1613

Narayanan, D., Turk, M., Feldmann, R., et al. 2015, Natur, 525, 496

Noguchi, M. 1998, Natur, 392, 253

Oklopcic, A., Hopkins, P. F., Feldmann, R., et al. 2016, MNRAS, in press (arXiv:1603.03778)

Oteo, I., Ivison, R. J., Dunne, L., et al. 2016, ApJ, 827, 34

Rich, J. W., de Blok, W. J. G., Cornwell, T. J., et al. 2008, AJ, 136, 2897

Sakamoto, K., Wang, J., Wiedner, M. C., et al. 2008, ApJ, 684, 957

Sanders, D. B., \& Mirabel, I. F. 1996, ARA\&A, 34, 749

Scoville, N., Murchikova, L., Walter, F., et al. 2016, ApJ, submitted (arXiv:1605.09381)

Shapiro, K. L., Genzel, R., Foerster Schreiber, N. M., et al. 2008, ApJ, 682, 231

Simpson, J. M., Smail, I., Swinbank, A. M., et al. 2015a, ApJ, 807, 128

Simpson, J. M., Smail, I., Swinbank, A. M., et al. 2015b, ApJ, 799, 81

Simpson, J. M., Swinbank, A. M., Smail, I., et al. 2014, ApJ, 788, 125

Smolčić, V., Aravena, M., Navarrete, F., et al. 2012, A\&A, 548, A4

Solomon, P. M., Rivolo, A. R., Barrett, J., \& Yahil, A. 1987, ApJ, 319, 730

Solomon, P. M., \& Vanden Bout, P. A. 2005, ARA\&A, 43, 677

Spergel, D. N., Verde, L., Peiris, H. V., et al. 2003, ApJS, 148, 175

Spergel, D. N., Bean, R., Dore, O., et al. 2007, ApJS, 170, 377

Steidel, C. C., Shapley, A. E., Pettini, M., et al. 2004, ApJ, 604, 534

Swinbank, A. M., Chapman, S. C., Smail, I., et al. 2006, MNRAS, 371, 465

Swinbank, A. M., Papadopoulos, P. P., Cox, P., et al. 2011, ApJ, 742, 11

Swinbank, A. M., Simpson, J. M., Smail, I., et al. 2014, MNRAS, 438, 1267

Swinbank, A. M., Smail, I., Chapman, S. C., et al. 2010a, MNRAS, 405, 234

Swinbank, A. M., Sobral, D., Smail, I., et al. 2012, MNRAS, 426, 935

Swinbank, A. M., Smail, I., Longmore, S., et al. 2010b, Natur, 464, 733

Tacconi, L. J., Genzel, R., Neri, R., et al. 2010, Natur, 463, 781

Tacconi, L. J., Neri, R., Genzel, R., et al. 2013, ApJ, 768, 74

Wang, S. X., Brandt, W. N., Luo, B., et al. 2013, ApJ, 778, 179

Wang, W.-H., Cowie, L. L., Barger, A. J., \& Williams, J. P. 2011, ApJL, 726, L18

Weiß, A., Kovacs, A., Coppin, K., et al. 2009, ApJ, 707, 1201

Yan, H., \& Ma, Z. 2016, ApJL, 820, L16

Younger, J. D., Fazio, G. G., Wilner, D. J., et al. 2008, ApJ, 688, 59 